\title{
ON DISTORTION IN PSEUDO-CONFORMAL MAPPING
}

\author{
BY \\ STEFAN BERGMAN AND D. C. SPENCER
}

\section{INTRODUCTION}

1.1. Suppose that the functions $w_{k}\left(z_{1}, z_{2}\right), k=1,2\left({ }^{1}\right)$, are regular in a four-dimensional domain $\mathfrak{B}_{1}$ of the complex variables $z_{1}, z_{2}$. The transformation $\mathbf{w}$ of $\mathfrak{B}_{1}$ into a domain $\mathfrak{B}_{2}$ by a pair of functions $w_{k}$, for which $\partial\left(w_{1}, w_{2}\right) / \partial\left(z_{1}, z_{2}\right)$ does not vanish identically, is called a PT (pseudo-conformal transformation). We are here concerned with general PT's in which the mapping is not necessarily one-one with respect to the schlicht space of the variables. Suppose that $\mathscr{B}_{1}$ is a fixed schlicht domain in the space $\left(z_{1}, z_{2}\right)$, which contains the point $(0,0)$ in its interior. Let

$$
\mathcal{C}=\mathcal{C}\left(\mathfrak{B}_{1}\right)
$$

be some specified subfamily of the family of domains $\mathfrak{B}_{2}$ obtainable from $\mathfrak{B}_{1}$ by PT's w which satisfy a certain normalization hypothesis at $(0,0)$; and let

$$
M=M\left(\mathfrak{B}_{2}\right)
$$

be the euclidean measure of a geometrical object defined for each $\mathfrak{B}_{2}$ of $\mathcal{C}\left({ }^{2}\right)$. The problem of the bounds between which $M\left(\mathfrak{B}_{2}\right)$ may vary for $\mathfrak{B}_{2} \in \mathcal{C}\left(\mathfrak{B}_{1}\right)$ forms an important chapter in the theor: $\left({ }^{3}\right)$.

In this paper we give inequalities for the volume of a subdomain $\mathfrak{B}_{2}^{\dagger}$ of $\mathfrak{B}_{2}$, where

$$
\mathfrak{B}_{2}^{\dagger}=S\left(\mathfrak{B}_{2}\right)
$$

is derived from $\mathfrak{B}_{2}$ by the following geometrical operation. We cut $\mathfrak{B}_{2}$ with the plane arg $w_{k}=\psi_{k}=$ constant, the intersection $\Im_{2}^{2}\left(\psi_{1}, \psi_{2}\right)$ being a plane set which consists in general of many disconnected components. We suppose that $\mathbf{w}(0,0)=(0,0)$; we denote by $\mathfrak{S}_{2}^{\dagger 2}\left(\psi_{1}, \psi_{2}\right)$ that connected component of the intersection of $\mathfrak{B}_{2}$ with arg $w_{k}=\psi_{k}$ for which $w(0,0)$ is a boundary point $\left({ }^{4}\right)$, and define $\mathfrak{B}_{2}^{\dagger}=\mathbf{S}\left(\mathfrak{B}_{2}\right)$ to be the domain of which the intersection with every

Presented to the Society, September 11, 1940; received by the editors November 25, 1940.

(1) To avoid endless repetition, we shall omit the statement " $k=1,2$ " hereafter. Whenever the index $k$ occurs, it is to have the values 1 and 2 .

(2) For example, $M$ may be the distance of the boundary of $\mathfrak{B}_{2}$ from the origin, or the volume of $\mathfrak{B}_{2}$.

(3) As it does in conformal mapping. See also Bergman [4, chap. 5] and [6, pp. 3-7]. The numbers in brackets refer to the bibliography, p. 162.

(4) Since in general the domain $\mathfrak{B}_{2}$ is not schlicht, there may exist points $(0,0)$ lying in other sheets which are images of points $\left(z_{1}, z_{2}\right)$ different from $(0,0)$. For a more detailed description of $\widetilde{S}_{2}^{\dagger 2}\left(\psi_{1}, \psi_{2}\right)$ see $\$ 7$. 
plane arg $w_{k}=\psi_{k}, 0 \leqq \psi_{k} \leqq 2 \pi$, is (with exception of a set of measure zero) $\Im_{2}^{\dagger 2}\left(\psi_{1}, \psi_{2}\right)$. (Figure 1 is a schematic diagram in which the quadrant arg $w_{k}=\psi_{k}$ is replaced by the half-line arg $w=\psi$, and represents the construction in one variable.) We obtain an inequality for the volume of $\mathfrak{B}_{2}^{\dagger}$ which depends: (i) on the initial domain $\mathfrak{B}_{1}\left({ }^{5}\right)$; (ii) on an average over $\mathfrak{B}_{1}$ of the

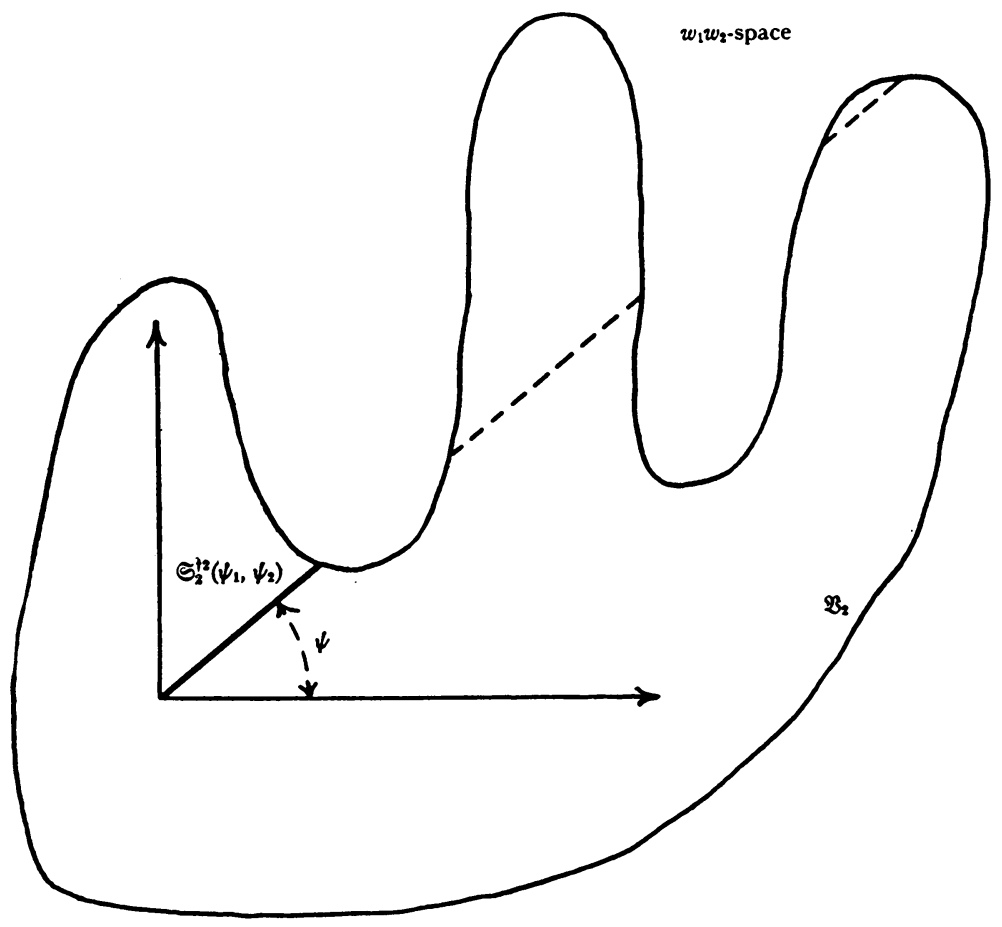

FIG. 1

first partial derivatives of $w_{k}$ (see (5.12)); and (iii) on the "mean valency" of $\mathfrak{B}_{2}^{\dagger}$-an hypothesis which, in the case of one complex variable, is the generalization of the notion of $p$-valency $\left(^{6}\right)$. All PT's of the given fixed domain $\mathfrak{B}_{1}$ may be arranged in classes $\mathcal{C}$ according to their "mean valency," and the inequality for the volume of $\mathfrak{B}_{2}^{\dagger}$ then depends on $\mathfrak{B}_{1}, \mathcal{C}$, and the average indicated in (ii).

Finally we should like to point out that the generalization of our results to $n$ variables, $n>2$, would involve no essentially new ideas.

(5) We take for $\mathfrak{B}_{1}$ the unit bicylinder, but an inequality analogous to the one given in this paper can be found for any domain $\mathfrak{B}_{1}$, and by the same general methods. However, the hypotheses of the theorem would have to be changed accordingly. Concerning other distortion theorems, see Bergman [4, pp. 124-158] and [5, chap. 9]. [1].

(6) The idea of mean valency in one variable was first introduced by Spencer in his paper 


\section{Notation}

2.1. Manifolds will be denoted by German letters, the superscript denoting the dimension of the manifold. However, we omit the superscript if the manifold is four-dimensional or a point. In operations on sets we use the customary symbols $\left({ }^{7}\right)$. For example, $\mathfrak{E}_{1}^{n} \cdot \mathfrak{E}_{2}^{m}$ denotes the common part (intersection) of $\mathfrak{E}_{1}^{n}$ and $\mathfrak{F}_{2}^{m}, \mathfrak{E}_{1}^{m} \times \mathfrak{E}_{2}^{n}$ the topological product of the two sets, $\mathfrak{a}^{n} \in \mathfrak{F}^{m}$ means that " $\mathfrak{a}^{n}$ is contained in $\mathcal{E}^{m}$," and so on.

We use the symbol $\delta$ to denote logical summation over a set,--for example

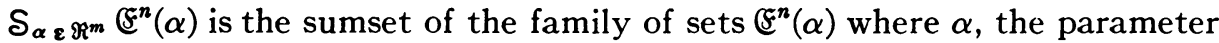
of the family, runs through a set $\Re^{m}$. By E [ ...] we mean the set of points the coordinates of which satisfy the conditions indicated in the brackets. However, the intersection of a manifold $\xi^{n}$ with $\mathrm{E}[\cdots]$ will be denoted $\mathbb{E}^{n} \cdot[\cdots]$. By $\mathrm{V}(\mathfrak{B})$ we denote the volume of a domain $\mathfrak{B}$, by $\mathrm{A}\left(\mathfrak{B}^{2}\right)$ the area of $\mathfrak{B}^{2}$.

In this paper we shall consider the PT's of manifolds of the $z_{1} z_{2}$-space, $z_{k}=x_{k}+i y_{k}$, into manifolds of the $w_{1} w_{2}$-space, $w_{k}=u_{k}+i v_{k}$, where $x_{1}, y_{1}, x_{2}, y_{2}$, and $u_{1}, v_{1}, u_{2}, v_{2}$, are the Cartesian coordinates of the respective spaces. The element of volume in the $z_{1} z_{2}$-space will be denoted by $d \omega_{z}$, in the $w_{1} w_{2}$-space by $d \omega_{w}$. Manifolds of the $z_{1} z_{2}$-space will be denoted by (German) letters with subscript 1 , and those of the $w_{1} w_{2}$-space will be denoted by letters with subscript 2 . Let $C\left(\alpha_{1}, \alpha_{2}, \alpha_{3}, \alpha_{4}\right)$ be some coordinate system in four-dimensional space. Given a manifold $\mathfrak{M}^{n}$, we define the projection of $\mathfrak{M}^{n}$ on $C_{1}\left(\alpha_{p}, \cdots, \alpha_{q}\right)$, $\mathbf{P}_{\alpha_{p} \cdots \alpha_{q}}\left(\mathfrak{M}^{n}\right)$ say, as the totality of number sets $\left(A_{p}, \cdots, A_{q}\right)$ for which $\left(A_{1}, A_{2}, A_{3}, A_{4}\right)$ are the coordinates of some point of $\mathfrak{M}^{n}$.

Finally we shall say that a function $f\left(z_{1}, z_{2}\right)$ is regular in $\mathfrak{M}^{n}, n \leqq 3$, if there exists a four-dimensional manifold containing $\mathfrak{M}^{n}$ in which $f$ is regular. In particular we shall say that a function $f\left(\sigma_{1}, \sigma_{2}\right)$ is an analytic function of two real variables $\sigma_{1}, \sigma_{2}$, regular in a domain $\mathfrak{F}^{2}$, if it can be developed in a power series $\sum a_{m n}\left(\sigma_{1}-\sigma_{1}^{(0)}\right)^{m}\left(\sigma_{2}-\sigma_{2}^{(0)}\right)^{n}$ at any point $\left(\sigma_{1}^{(0)}, \sigma_{2}^{(0)}\right)$ of $\mathfrak{F}^{2}$, the series converging absolutely and uniformly in some neighborhood of $\left(\sigma_{1}^{(0)}, \sigma_{2}^{(0)}\right)$.

\section{RESULTS FOR FUNCTIONS OF ONE VARIABLE}

3.1. The result which we prove is a generalization to two complex variables of a theorem of Golusin [1], which in turn is a generalization of the lemma of Schwarz.

Suppose that $w(z)$ is regular for $|z|<1$, that $w(0)=0,\left|w^{\prime}(0)\right|=1$, and that $\mathfrak{B}_{2}^{2}$ is the map of $\mathrm{E}[|z|<1]$ by $\mathbf{w}=[w=w(z)]$. Then $\mathrm{A}\left(\mathfrak{B}_{2}^{2}\right)$ (multiplycovered regions being counted multiply) is not less than $\pi$. Golusin's theorem states that not only $\mathrm{A}\left(\mathfrak{B}_{2}^{2}\right)$, but also $\mathrm{A}\left(\mathfrak{B}_{2}^{\dagger 2}\right)$, is at least equal to $\pi$, where the domain $\mathfrak{B}_{2}^{\dagger 2}$ is the two-dimensional analogue of the domain $\mathfrak{B}^{\dagger}$ defined above (see Figure 1). A more precise version of Golusin's theorem was given by Bermant [1] and lately a still more precise one by Spencer [2 ].

( ${ }^{7}$ See for example Hausdorff, Mengenlehre, 2d edition, 1928, Section 1. 
The final form of the results may be stated as follows:

Suppose that $w(z)$ is regular for $\mathrm{E}[|z|<1]$, that $w(0)=0,\left|w^{\prime}(0)\right|=1$, and let $\mathfrak{B}_{2}^{\dagger 2}$ be the portion of $\mathfrak{B}_{2}^{2}$ which is visible to an eye placed at $w(0)$ if all boundary elements of $\mathfrak{B}^{2}$ are opaque. Then

$$
\mathrm{A}\left[\mathrm{w}^{-1}\left(\mathfrak{B}_{2}^{\dagger 2}\right)\right] \cdot \mathrm{A}\left(\mathfrak{B}_{2}^{\dagger 2}\right) \geqq \pi^{2},
$$

with equality only if $w=z\left({ }^{8}\right)$.

Now the generalization of these results to two variables is not immediate; we require certain new ideas and methods, in particular the following two concepts: (i) Four-dimensional "mean valency," and a new representation for the volume of a four-dimensional (in general multiply-covered) domain (see §4). Mean valency here assumes an interesting form, which puts the general concept of valency in a new light, even in one variable. (ii) A new kind of area, " $B$-area." If, in passing from conformal transformations to PT's, ordinary area is replaced by volume, length of curves by $B$-area of surfaces, a generalization is obtained, involving $B$-area and volume, of inequalities between length and area (see Bergman [4, Chap 5, §6, pp. 147-149]), and this method is adopted in $\S 8$.

\section{Mean valency}

4.1. Given a space $\mathfrak{U}^{2 n}=\mathrm{E}\left[\left|w_{\mathrm{H}}\right|<\infty, \mathrm{H}=1,2, \cdots, n\right]$ we first choose a suitable coordinate system $\mathfrak{W}_{\text {in }} \mathfrak{U}^{2 n}$, and then some particular one of the coordinates, $\lambda=\lambda(w)$, say. If $\mathfrak{B}^{2 n}$ is an arbitrary (in general multiply-covered) domain, we denote by $\mathfrak{B}^{2 n}(\Lambda)$ the domain $\mathfrak{B}^{2 n} \cdot[\lambda<\Lambda]$, that is, the subdomain of $\mathfrak{B}^{2 n}$ for which $0 \leqq \lambda<\Lambda$. Suppose now that $\mathfrak{B}_{0}^{2 n}$ is a given domain. Then we say that $\mathfrak{B}^{2 n}$ is mean $p$-valent with respect to $\mathfrak{B}_{0}^{2 n}$ in the direction $\lambda$ if

$$
\mathrm{V}\left[\mathfrak{B}^{2 n}(\Lambda)\right] \leqq p \mathrm{~V}\left[\mathfrak{B}_{0}^{2 n}(\Lambda)\right]
$$

for all $\Lambda>0$, where $p$ is a positive real number $\left({ }^{9}\right)$. Since in this paper we shall be concerned with only one domain $\mathfrak{B}_{0}^{2 n}$, and with fixed direction of measure $\lambda$ in a fixed system of coordinates, we shall say simply that $\mathfrak{B}^{2 n}$ is mean $p$-valent. For example, suppose that $n=1$, and that we choose for $\mathscr{W}$ the polar co-

$\left.{ }^{8}\right) \mathbf{w}^{-1}\left(\mathfrak{B}_{2}^{\dagger}\right)$ and $\mathfrak{B}_{2}^{\dagger}$ are both schlicht domains which contain the points $z=0, w=0$, re spectively (but in two variables $\mathfrak{B}_{2}^{\dagger}$ is not necessarily schlicht). $\mathfrak{B}_{2}^{\dagger 2}$ is a "star-like" domain (the "star" of $\mathfrak{B}_{2}^{2}$ with respect to the point $w(0)$ ). Any point of $\mathfrak{B}_{2}^{+2}$ can be reached by starting at $w(0)=0$ and travelling outward along a radius.

If $\mathfrak{B}^{2}$ is itself a "star" domain, then $\mathfrak{B}^{+2}=\mathfrak{B}^{2}$; the difference between the areas of $\mathfrak{B}^{2}$ and $\mathfrak{B}^{+2}$ is in a certain sense inversely as the "star-likeness" of $\mathfrak{B}^{2}$.

(9) We could, of course, define mean valency without introducing the domain $\mathfrak{B}_{0}^{2 n}$, merely replacing the right-hand member of $(4.1 .1)$ by a function of $\Lambda$; but the geometrical interpretation seems to us desirable here.

When $n=1, \mathrm{~V}$ becomes area, which we denote by $\mathrm{A}$. 
ordinates $\lambda, \psi\left({ }^{10}\right)$, so that $w=\lambda e^{i \psi}$. If then we take $\mathfrak{B}_{0}^{2}$ to be the whole plane, that is

$$
\mathfrak{B}_{0}^{2}=\mathrm{E}[|w| \leqq 1]+\mathrm{E}[|w| \geqq 1],
$$

the inequality (4.1.1) becomes the hypothesis of mean $p$-valency in one variable (see Spencer [1]).

In the case of $\mathfrak{U}^{4}$ we write $w_{k}=r_{k} e^{i \psi_{k}}$, and define $\lambda$ and $\mu$ by the equation

$$
\mu+i \lambda=\left(r_{1}+i r_{2}\right)^{2} .
$$

We take now for $\mathscr{W}$ the coordinates $\left.{ }^{(11}\right) \lambda, \mu, \psi_{1}, \psi_{2}$. Next, in analogy with (4.1.2), we take

$$
\mathfrak{B}_{0}=\mathrm{E}\left[\left|w_{k}\right| \leqq 1\right]+\mathrm{E}\left[\left|w_{k}\right| \geqq 1\right] .
$$

The coordinate system $\lambda, \mu, \psi_{1}, \psi_{2}$, and standard domain $\mathfrak{B}_{0}$ defined by (4.1.3) and (4.1.4), respectively, will be used throughout this paper.

4.2. We now formulate these definitions analytically, and, for purposes of comparison, we first formulate the hypothesis in one variable.

Let $\left({ }^{12}\right) \mathrm{n}(\lambda, \psi)=\mathrm{n}_{\mathfrak{B}_{2}}(\lambda, \psi)$ be the number of times $\mathfrak{B}^{2}$ covers the point $(\lambda, \psi)$ of the schlicht space. We define

$$
\mathrm{p}_{\mathfrak{B}^{2}}(\lambda)=(1 / 2 \pi) \int_{-\pi}^{\pi} \mathrm{n}(\lambda, \psi) d \psi
$$

Then

$$
\mathrm{A}\left[\mathfrak{B}^{2}(\Lambda)\right]=\int_{0}^{\Lambda} \mathrm{p}(\lambda) d\left(\pi \lambda^{2}\right)
$$

In particular, taking $\mathfrak{B}^{2}=\mathfrak{B}_{0}^{2}$, we have $p(\lambda)=\mathrm{p}_{\mathfrak{B}_{0}^{2}}(\lambda)=1$ for all $\lambda$, and so

$$
\mathrm{A}\left[\mathfrak{B}_{0}^{2}(\Lambda)\right]=\int_{0}^{\Delta} d\left(\pi \lambda^{2}\right) .
$$

Substituting from (4.2.3) and (4.2.2) into (4.1.1), the hypothesis of mean $p$-valency in one variable may be expressed in the form

$$
\int_{0}^{\Delta} \mathrm{p}(\lambda) d\left(\pi \lambda^{2}\right) \leqq p \int_{0}^{\Delta} d\left(\pi \lambda^{2}\right)=p \pi \Lambda^{2}
$$

4.3. In the space of two variables we choose the coordinate system defined above by (4.1.3), and take for $\mathfrak{B}_{0}$ the domain (4.1.4). We let $n\left(\lambda, \mu, \psi_{1}, \psi_{2}\right)$ $=\mathrm{n}_{\mathfrak{B}}\left(\lambda, \mu, \psi_{1}, \psi_{2}\right)$ be the number of times that $\mathfrak{B}$ covers the point $\left(\lambda, \mu, \psi_{1}, \psi_{2}\right)$ of the schlicht space, and define

(10) $\lambda$ will be used throughout to denote the coordinate which occurs above.

(11) These coordinates assume for some purposes a role similar to that of the polar coordinates $\lambda, \psi$ in one variable. See Bergman $[3,83]$.

(12) We omit the subscript $\mathfrak{B}^{2}$ when it is clear on what domain the functions $n, p, \cdots$ depend. 
Then

$$
\begin{aligned}
\mathrm{N}_{\mathfrak{B}}\left(\lambda, \psi_{1}, \psi_{2}\right) & =(1 / 4) \int_{-\infty}^{\infty} \mathrm{n}\left(\lambda, \mu, \psi_{1}, \psi_{2}\right)\left(\lambda^{2}+\mu^{2}\right)^{-1 / 2} d \mu, \\
\mathrm{p}_{\mathfrak{B}}(\lambda) & =\left(1 / 4 \pi^{2}\right) \int_{-\pi}^{\pi} \int_{-\pi}^{\pi} \mathrm{N}_{\mathfrak{B}}\left(\lambda, \psi_{1}, \psi_{2}\right) d \psi_{1} d \psi_{2} .
\end{aligned}
$$

$$
\mathrm{V}[\mathfrak{B}(\Lambda)]=\int_{0}^{\Lambda} \mathrm{p}(\lambda) d\left(\pi^{2} \lambda^{2}\right)
$$

In particular, if we take $\mathfrak{B}=\mathfrak{B}_{0}$ (cf. (4.1.4)), we have $\mathrm{p}(\lambda)=\mathrm{p}_{\mathfrak{B}_{0}}(\lambda)$ $=(1 / 2)|\lg (2 / \lambda)|$, and so

$$
\mathrm{V}\left[\mathfrak{B}_{0}(\Lambda)\right]=(p / 2) \int_{0}^{\Lambda}|\lg (2 / \lambda)| d\left(\pi^{2} \lambda^{2}\right) .
$$

Substituting from (4.3.3) and (4.3.4) into (4.1.1), the hypothesis of mean $p$-valency in two variables becomes

$$
\int_{0}^{\Lambda} \mathrm{p}(\lambda) d\left(\pi^{2} \lambda^{2}\right) \leqq(p / 2) \int_{0}^{\Lambda}|\lg (2 / \lambda)| d\left(\pi^{2} \lambda^{2}\right) \quad(\Lambda>0) .
$$

REMARK. If $P$ is a point for which $\lambda=0$, we shall (for convenience) denote the coordinates of $P$ by $\left(0, \mu, \psi_{1}, \psi_{2}\right)$ even though $\psi_{1}$ or $\psi_{2}$ may be meaningless. For example, if $\mu>0$, we shall write $\mathrm{n}\left(0, \mu, \psi_{1}, \psi_{2}\right)$ for $\mathrm{n}\left(0, \mu, \psi_{1}\right)$.

4.4. A necessary and sufficient condition that $p$-valency should imply mean $p$-valency with respect to a domain $\mathfrak{B}_{0}$ is that

$$
\mathfrak{B}_{0}^{2 n}=\mathfrak{U}^{2 n} \text {. }
$$

The particular definition of mean $p$-valency (bicylindrical mean $p$-valency) selected above satisfies this condition in one, but not in two, variables. Since this definition carries with it certain important metrical properties, it is natural to expect that many theorems associated with valency in one variable will be true in two variables under a hypothesis of mean valency, but false under one of valency alone.

4.5. In this paper we shall be concerned with the hypothesis only when $p=1$. Suppose that $F(\Lambda)$ is a real function of $\Lambda$, defined for $\Lambda \geqq 0$, which satisfies the following two conditions:

$$
\begin{aligned}
& 0 \leqq \Lambda F(\Lambda) \leqq 1 \\
& \int_{0}^{\infty} F(\Lambda) d \Lambda \leqq 1 / 2 .
\end{aligned}
$$$$
(\Lambda>0)
$$

We shall say that a domain $\mathfrak{B}$ is mean one-valent with excess $\alpha$, or that $\mathfrak{B}$ belongs to the class $\mathcal{T}(\alpha), \alpha \geqq 0$, if there exists an $F(\Lambda)$ satisfying (4.5.1) and (4.5.2) and such that, for all $\Lambda>0$,

$$
\int_{0}^{\Delta} \mathrm{p}_{\mathfrak{B}}(\lambda) d\left(\pi^{2} \lambda^{2}\right) \leqq(1 / 2) \int_{0}^{\Delta}|\lg (2 / \lambda)| d\left(\pi^{2} \lambda^{2}\right)+\alpha \Lambda^{3} F(\Lambda) .
$$


5.1. We write

\section{Statement OF THE THEOREM}

$$
z_{k}=\rho_{k} e^{i \theta_{k}}, \quad d \omega_{z}=\rho_{1} \rho_{2} d \rho_{1} d \rho_{2} d \theta_{1} d \theta_{2},
$$

and denote the unit bicylinder $\mathrm{E}\left[\left|\boldsymbol{z}_{k}\right|<1\right]$ by $\mathfrak{B}_{1}$. By $\mathfrak{B}^{\dagger}$ we mean the domain obtained from a domain $\mathfrak{B}$ by the geometrical operation discussed in the introduction $(\$ 1)\left({ }^{13}\right)$.

Theorem. Suppose that the PT

$$
\mathbf{w}: \quad w_{k}=w_{k}\left(z_{1}, z_{2}\right) \equiv a_{2-k, k-1}^{(k)} z_{k}+z_{1}^{3-k} z_{2} f_{k}\left(z_{1}, z_{2}\right),
$$

where $\left|a_{2-k, k-1}^{(k)}\right|=1$, and where $f_{k}\left(z_{1}, z_{2}\right)$ is regular in $\mathfrak{B}_{1}$, maps $\mathfrak{B}_{1}$ on a domain $\mathfrak{B}_{2}$. Then (i) if $\mathfrak{B}_{2}^{\dagger} \in \mathcal{G}\left(\alpha_{1}\right)$ [in particular if $\mathfrak{B}_{2} \in \mathcal{T}\left(\alpha_{1}\right)$ ], and (ii) if

$$
\iiint \int_{w^{-1}\left(\mathfrak{B}_{2}^{\dagger}\right)} \sum_{k=1}^{2}\left[\frac{1}{\left|z_{k}\right|^{2}}\left|\frac{\partial w_{1}}{\partial z_{3-k}} \cdot \frac{\partial w_{2}}{\partial z_{3-k}}\right|\right] \frac{d \omega_{z}}{\left|w_{1} w_{2}\right|} \leqq \alpha_{2}
$$

(in particular if $\iiint \int_{\mathfrak{B}_{1}} \cdots \leqq \alpha_{2}$ ), we have

$$
\mathrm{V}\left[\mathrm{w}^{-1}\left(\mathfrak{B}_{2}^{\dagger}\right)\right] \cdot \mathrm{V}\left(\mathfrak{B}_{2}^{\dagger}\right) \geqq K\left(\alpha_{1}, \alpha_{2}\right)
$$

where

$$
K\left(\alpha_{1}, \alpha_{2}\right)=(\pi / 4)^{4} \exp \left[-\frac{4 \alpha_{1}+\alpha_{2}}{\pi^{2} \lg 2}\right] .
$$

REMARK. We prove the theorem subject to the additional hypothesis that $\left.{ }^{14}\right) \mathrm{n}_{\mathfrak{B}_{2}}\left(0, \mu, \psi_{1}, \psi_{2}\right) \leqq 1$, but indicate in $\$ 9$ how this restriction may be removed. However, since the removal of the condition involves only technical complications and no new ideas, we give only a sketch.

5.2. Let $\mathcal{C}$ be the class of domains $\mathfrak{B}_{2}$ arising from the bicylinder( $\left.{ }^{15}\right) \mathfrak{B}_{1}$ by PT's w normalized at $(0,0)$. If $P$ is a point of $\mathfrak{B}_{2}$, we may take for the coordinates of $P$ the coordinates of the point $P^{\prime}=w^{-1}(P)$, and thus define in $\mathfrak{B}_{2}$ a new system of coordinates $K\left(\mathfrak{B}_{2}\right)$, say. The intersection $\mathfrak{S}_{2}^{\dagger 2}\left(\psi_{1}, \psi_{2}\right)$ defined in $\$ 1$ has the property that its area (multiply-covered portions being counted multiply) is the same whether measured in the $A$ - or $B$-sense ( $A$ is ordinary area; for the definition of $B$-area see Bergman [2], or $\$ 8.1$ ). The integral on the left-hand side of (5.1.2) is a measure of the invariance of this property to the change of coordinates, and (5.1.2) states that the average with respect to $\left(\psi_{1}, \psi_{2}\right)$ of the difference between the $A$ - and $B$-areas of $\mathfrak{S}_{2}^{\dagger 2}\left(\psi_{1}, \psi_{2}\right)$ in the new system $K\left(\mathscr{B}_{2}\right)$ does not exceed $\alpha_{2}$.

(13) For a more detailed description of $\mathfrak{B}^{\dagger}$ we refer the reader to $\$ 7$.

(14) For the definition of $n$ see $\$ 4$.

(i5) The bicylinder $\mathfrak{B}_{1}$ is the "representative domain" of $\mathcal{C}$ with respect to $(0,0)$ see Berg$\operatorname{man}[4$, chap. 7 , pp. 215-230] and [5, chap. 10]). 
5.3. By the theorem in one variable cited in $\S 3$, we see that the result of the theorem is true with $K=\pi^{4}$ when $w$ is a product PT. In the above theorem we suppose that all but one of the coefficients which border the matrices of coefficients of $w_{1}$ and $w_{2}$ are zero, and this hypothesis in itself $\left({ }^{16}\right)$ excludes from consideration all product PT's except the trivial rotation $w_{k}=a_{2-k, k-1}^{(k)} z_{k}$. Although product PT's (in which only the coefficients bordering the matrices of $w_{1}$ and $w_{2}$ appear) are a small class compared to the class of PT's admitted by our hypothesis (even for fixed $\alpha_{1}, \alpha_{2}$ ), nevertheless the question arises as to why product PT's are not a special case of our theorem. The answer is that the inclusion of "product-like" PT's would involve nothing essentially new, but would require two mutually exclusive sets of hypotheses in our theorem.

\section{Preliminary Remarks Concerning the proof}

6.1. The proof is divided into two parts-a geometrical part ( $\$ 7)$, and an analytical part (\$8). In order not to interrupt the argument later, we sum up here certain facts to which we shall constantly refer.

6.2. First, we may assume that the functions $w_{k}$ are regular in the closed bicylinder $\mathfrak{D}_{1}\left(\mathfrak{D}_{1}\right.$ is the closure of $\left.\mathfrak{B}_{1}\right)$; therefore in some domain which contains $\mathfrak{D}_{1}$. For, having proved the theorem in this special case, we can apply it to the functions

$$
\rho^{-1} w_{k}\left(\rho z_{1}, \rho z_{2}\right)=a_{2-k, k-1}^{(k)} z_{k}+z_{1}^{3-k} z_{2} \rho 2 f_{k}\left(\rho z_{1}, \rho z_{2}\right)
$$

where $\rho<1$, and then let $\rho \rightarrow 1$. In particular, we may assume that $f_{1}$ and $f_{2}$ are bounded in $\mathfrak{D}_{1}$.

Secondly, from the hypothesis that the PT is schlicht on $\left|z_{k}\right|=0$, it now follows that there exists a $\delta$ such that the mapping is schlicht for $\left|z_{1} z_{2}\right|<\delta$. Assuming, therefore, that $\epsilon$ is sufficiently small, we see that the pair of inverse functions $z_{k}\left(w_{1}, w_{2}\right)$ have the form

$$
z_{k}\left(w_{1}, w_{2}\right)=w_{k} / a_{2-k, k-1}^{(k)}+w_{1}^{3-k} w_{2}^{k} g_{k}\left(w_{1}, w_{2}\right),
$$

where the $g_{k}$ are both regular in the portion of $\mathfrak{D}_{2}\left(\mathfrak{D}_{2}\right.$ is the closure of $\left.\mathfrak{B}_{2}\right)$ for which either $\left|w_{1}\right|<\epsilon$, or $\left|w_{2}\right|<\epsilon$. A particular consequence of (6.2.2) is that, if $\lambda \leqq \lambda_{0}$, points $\left(w_{1}, w_{2}\right)$ of $\mathfrak{D}_{2}$ for which $\left|w_{1} w_{2}\right|=\lambda$, are transformed into points $\left(z_{1}, z_{2}\right)$ which satisfy

$$
\begin{aligned}
\left|z_{1} z_{2}\right| & =\left|w_{1}+a_{10}^{(1)} w_{1}^{2} w_{2} g_{1}\right| \cdot\left|w_{2}+a_{01}^{(2)} w_{1}^{2} w_{2}^{2} g_{2}\right| \\
& =\left|w_{1} w_{2}\right|+O\left(\left|w_{1} w_{2}\right|^{2}\right)=\lambda+O\left(\lambda^{2}\right),
\end{aligned}
$$

and conversely.

(16) Furthermore, product PT's do not in general satisfy the mean-valency restrictions of our theorem when $\alpha_{1}$ is finite (but they satisfy (5.1.2) with $\alpha_{2}=0$ ). 


\section{Proof. Geometrical part}

7.1. Throughout this section we assume that the PT's which we consider satisfy not only the hypotheses of the theorem, but also the additional hypotheses of $\S 6$. In particular, therefore, we assume that the functions $w_{k}$ are regular for $\mathrm{E}\left[\left|z_{k}\right| \leqq \mathrm{P}_{k}\right]$, where $\mathrm{P}_{k}>1$. Such a PT transforms the (closed) bicylinder

$$
\mathfrak{D}_{1}\left(\mathbf{P}_{k}\right)=\mathrm{E}\left[\left|z_{k}\right| \leqq \mathrm{P}_{k}\right]
$$

into a domain $\mathfrak{D}_{2}\left(\mathrm{P}_{k}\right)$ imbedded in a many-sheeted Riemannian space. At each point $P$ of $\mathfrak{B}_{1}\left(\mathrm{P}_{k}\right)=\mathrm{E}\left[\left|z_{k}\right|<\mathrm{P}_{k}\right]$ at which

$$
J\left(z_{1}, z_{2}\right)=\partial\left(w_{1}, w_{2}\right) / \partial\left(z_{1}, z_{2}\right) \neq 0
$$

the PT is locally schlicht: that is to say, the different sheets are not connected in a neighborhood of $\mathbf{w}(P)$. The surface $\mathfrak{B}_{2}^{2}=\mathbf{w}\left(\mathfrak{B}_{1}^{2}\right)$, where

$$
\mathfrak{B}_{1}^{2}=\mathrm{E}\left[J\left(z_{1}, z_{2}\right)=0\right],
$$

is the "branch surface" of the Riemannian space in which $\mathfrak{D}_{2}\left(\mathbf{P}_{k}\right)$ lies. We divide the points of $\mathfrak{B}_{2}^{2}$ into two classes: (i) branch points of the first kind, which are transforms of points $\left(z_{1}, z_{2}\right)$ at which $J\left(z_{1}, z_{2}\right)$ has only one prime factor (which may, however, be raised to a power higher than the first); and (ii) branch points of the second kind, which correspond to points $\left(z_{1}, z_{2}\right)$ where $J\left(z_{1}, z_{2}\right)$ has more than one prime factor $\left.{ }^{17}\right)$. Since $J$ has only a finite number of factors $\left(z_{2}-a_{2}^{(p)}\right)$, we can, by the Weierstrass preparation theorem and the Heine-Borel covering theorem, cover $\mathfrak{B}_{1}\left(\mathrm{P}_{k}\right)$ with finitely many domains $\mathfrak{S}_{1, j}, j=1,2, \cdots, n$, such that $\left({ }^{18}\right)$, in each $\mathfrak{G}_{1, j}$

$$
J\left(z_{1}, z_{2}\right)=z_{2}^{\mu} \prod_{\mathbf{H}}\left[z_{1}-\alpha_{\mathbf{H}}\left(z_{2}\right)\right]^{\lambda_{\mathbf{H}}} \Omega\left(z_{1}, z_{2}\right)
$$

where $\Omega\left(z_{1}, z_{2}\right)$ is regular and nonvanishing in $\mathcal{G}_{1, p}$, and where the $\alpha_{\mathrm{H}}\left(z_{2}\right)$ are algebroid functions $\left({ }^{19}\right)$.

The function

$$
\Delta\left(z_{2}\right)=\prod_{\mathbf{H}, \rho ; \mathbf{H} \neq \rho}\left[\alpha_{\mathbf{H}}\left(z_{2}\right)-\alpha_{\rho}\left(z_{2}\right)\right]^{2},
$$

being a regular function of $z_{2}$, vanishes at only a finite number of points $a_{2}^{(p)}$. Since there are only finitely many points $\left(a_{1}^{(p)}, a_{2}^{(p)}\right), a_{1}^{(p)}=\alpha_{\mathrm{H}}\left(a_{2}^{(p)}\right)$ there are

(17) See Osgood [1, chap. $2, \S 20$, p. 111]. With exception of finitely many points $\left(z_{1}^{(\nu)}, z_{2}^{(\nu)}\right)^{\prime}$ we can suppose that these factors have the form $\left[z_{1}-\alpha\left(z_{2}\right)\right]$.

(18) For the sake of brevity we suppose that the point in the neighborhood of which we consider $J\left(z_{1}, z_{2}\right)$ is the origin.

(19) A function $\alpha=\alpha(z)$ satisfying the equation $\alpha^{m}+g_{1}(z) \alpha^{m-1}+\cdots+g_{m}(z)=0$, where $g_{p}(z), p=1,2, \cdots, m$, are analytic functions of one complex variable regular in $\mathfrak{N}^{2}$, is said to be "algebroid in $\mathfrak{N}^{2}$." 
only finitely many branch points of the second kind and we can, therefore, with finitely many exceptions, write in the neighborhood of every point $\left(z_{1}^{(0)}, z_{2}^{(0)}\right)$ either

$$
J\left(z_{1}, z_{2}\right)=\left[z_{1}^{\prime}-\alpha\left(z_{2}^{\prime}\right)\right]^{\mu} \Omega\left(z_{1}^{\prime}, z_{2}^{\prime}\right),
$$

or

$$
J\left(z_{1}, z_{2}\right)=z_{2}^{\prime \mu} \Omega\left(z_{1}^{\prime}, z_{2}^{\prime}\right),
$$

where $z_{k}^{\prime}=z_{k}-z_{k}^{(0)}$, and where $\alpha\left(z_{2}^{\prime}\right)$ is a regular function of $z_{2}$. We remove from $\mathfrak{B}_{2}^{2}$ the branch points of the second kind, and denote the remaining surface by $\mathbb{E}_{2}^{2}$.

By the Heine-Borel theorem and the results of Osgood [1] we can cover every closed subdomain of $\mathbb{E}_{2}^{2}$ with finitely many neighborhoods $\mathfrak{\mho}_{2, p}$ such that to each $\mathfrak{F}_{2, p}$ there corresponds a uniformizing transformation

$$
\sigma_{1}=\left(w_{1}-H\left(w_{2}\right)\right)^{1 / m}, \quad \sigma_{2}=w_{2},
$$

or $\sigma_{1}=w_{1}, \sigma_{2}=\left(w_{2}-H\left(w_{1}\right)\right)^{1 / m}$, where $H$ is a suitably chosen analytic function of one complex variable, such that the PT

$$
\sigma_{k}=\sigma_{k}\left(z_{1}, z_{2}\right)
$$

is one-one in $\mathfrak{F}_{2, p}$.

7.2. We now derive certain trivial properties of $w$, in particular, that $w$ is a topological transformation. We write

$$
\begin{aligned}
\mathfrak{B}_{1, \epsilon}\left(\mathrm{P}_{k}\right)=\mathfrak{B}_{1}\left(\mathrm{P}_{k}\right) & -\int_{k=1}^{2} \mathrm{E}\left[\left|z_{k}\right| \leqq \epsilon\right] \\
& -\underset{\left(\zeta_{1}, \zeta_{2}\right) \mathfrak{E}_{1}{ }^{2}}{S_{2}} \mathrm{E}\left[\left|z_{1}-\zeta_{1}\right|^{2}+\left|z_{2}-\zeta_{2}\right|^{2} \leqq \epsilon^{2}\right],
\end{aligned}
$$

$$
\mathfrak{B}_{2, \epsilon}\left(\mathrm{P}_{k}\right)=\mathrm{w}\left[\mathfrak{B}_{1, \epsilon}\left(\mathrm{P}_{k}\right)\right] \text {. }
$$

Lemma 1. There exists a number $C$, depending only on $\mathrm{w}$ and $\epsilon$, such that we have the following inequality connecting the length $\mathrm{L}\left(\mathfrak{l}_{1}^{1}\right)$ of a curve $\mathfrak{l}_{1}^{1}$ in $\mathfrak{B}_{1, \epsilon}\left(\mathrm{P}_{k}\right)$ with the length of its transform $\mathfrak{l}_{2}^{1}=\mathbf{w}\left(\mathfrak{l}_{1}^{1}\right)$ :

$$
C^{-1} \mathrm{~L}\left(\mathfrak{l}_{2}^{1}\right) \leqq \mathrm{L}\left(\mathfrak{l}_{1}^{1}\right) \leqq C \mathrm{~L}\left(\mathfrak{l}_{2}^{1}\right) .
$$

In fact, let $\mathfrak{l}_{2}^{1}=\mathrm{E}\left[w_{k}=w_{k}(u), 0 \leqq u \leqq 1\right]$, where $w_{k}(u)$ is differentiable in $u$. Then

$$
\begin{aligned}
\left\{d \mathrm{~L}\left[\mathfrak{l}_{1}^{1}(u)\right]\right\}^{2} & =\left(\sum_{j=1}^{2}\left|\frac{d z_{j}}{d u}\right|^{2}\right) d u^{2}=\sum_{j=1}^{2}\left|\sum_{k=1}^{2} \frac{\partial z_{j}}{\partial w_{k}} \frac{\partial w_{k}}{\partial u}\right|^{2} d u^{2} \\
& \leqq\left[\sum_{j=1}^{2} \sum_{k=1}^{2}\left|\frac{\partial z_{k}}{\partial w_{j}}\right|^{2}\right] \cdot\left[\sum_{k=1}^{2}\left|\frac{\partial w_{k}}{\partial u}\right|^{2}\right] d u^{2}
\end{aligned}
$$


and analogously

$$
\left\{d \mathrm{~L}\left[l_{2}^{1}(u)\right]\right\}^{2} \leqq\left[\sum_{j=1}^{2} \sum_{k=1}^{2}\left|\frac{\partial w_{k}}{\partial z_{j}}\right|^{2}\right] \cdot\left[\sum_{k=1}^{2}\left|\frac{\partial z_{k}}{\partial u}\right|^{2}\right] d u^{2} .
$$

Setting

$$
C=\max \left[\underset{\left(w_{1}, w_{2}\right) \in \max _{2, \epsilon}\left(\mathbf{P}_{k}\right)}{ } \sum_{j=1}^{2} \sum_{k=1}^{2}\left|\frac{\partial z_{k}}{\partial w_{j}}\right|^{2}, \underset{\left(z_{1}, z_{2}\right) \in \mathfrak{W}_{1, \epsilon}\left(\mathbf{P}_{k}\right)}{\max } \sum_{j=1}^{2} \sum_{k=1}^{2}\left|\frac{\partial w_{k}}{\partial z_{j}}\right|^{2}\right],
$$

we have the lemma. Furthermore $\mathrm{L}\left(\mathfrak{l}_{2}^{1}\right) \leqq C \mathrm{~L}\left(\mathfrak{l}_{1}^{1}\right)$ throughout $\mathfrak{B}_{1}\left(\mathbf{P}_{k}\right)$; and $\mathrm{L}\left(\mathfrak{l}_{1}^{1}\right) \leqq C L^{*}\left(\mathfrak{l}_{2}^{1}\right)$ if $\mathfrak{l}_{1}^{1} \in \mathfrak{F}_{1, m}$, where $\mathrm{L}^{*}\left(\mathfrak{l}_{2}^{1}\right)$ denotes the length of $\mathfrak{l}_{2}^{1}$ in the $\sigma_{1} \sigma_{2}$-space (see (7.13)), and $\mathfrak{F}_{1, m}=\mathbf{w}^{-1}\left(\mathfrak{F}_{2, m}\right)$.

Since the transforms of the hyperspheres of radius $\epsilon$ and centers $\mathbf{w}^{-1}\left(w_{1}^{(\nu)}, w_{2}^{(\nu)}\right)$ lie in an arbitrarily small neighborhood of $\left(w_{1}^{(\nu)}, w_{2}^{(\nu)}\right)$, where $\left(w_{1}^{(\nu)}, w_{2}^{(\nu)}\right), \nu=1,2, \cdots, n$, are branch points of the second kind, we see that, if a sequence of points $P_{\mathrm{H}}$ tends to a limit $P_{0}$ in the $z_{1} z_{2^{-}}$(or $\left.w_{1} w_{2^{-}}\right)$space, then $\lim _{\mathrm{H} \rightarrow \infty} \mathbf{w}\left(P_{\mathrm{H}}\right)=\mathbf{w}\left(P_{0}\right)\left(\right.$ or $\left.\lim _{\mathrm{H} \rightarrow \infty} \mathbf{w}^{-1}\left(P_{\mathrm{H}}\right)=\mathbf{w}^{-1}\left(P_{0}\right)\right)$. Also

$$
\lim _{\epsilon \rightarrow 0} \mathrm{~V}\left[\mathfrak{B}_{2, \epsilon}\left(\mathbf{P}_{k}^{\prime}\right)\right]=\mathrm{V}\left[\mathfrak{B}_{2}\left(\mathbf{P}_{k}^{\prime}\right)\right] \quad\left(\mathbf{P}_{k}^{\prime}<\mathbf{P}_{k}\right) .
$$

7.3. We shall denote the transform of the bicylinder $\mathfrak{B}_{1}\left(\tau_{k}\right)=\mathrm{E}\left[\left|z_{k}\right|<\tau_{k}\right]$, $\tau_{k}<\mathrm{P}_{k}$, by $\mathfrak{B}_{2}\left(\tau_{k}\right)$. The boundary of $\mathfrak{B}_{2}\left(\tau_{k}\right)$ consists of the two hypersurfaces $\left({ }^{20}\right)$

$$
\begin{aligned}
& \mathfrak{G}_{21}^{3}\left(\tau_{k}\right)=\underset{0 \leqq \lambda \leqq 2 \pi}{\mathcal{S}} \mathrm{E}\left[w_{k}=w_{k}\left(\tau_{1} e^{i \lambda}, z_{2}\right),\left|z_{2}\right| \leqq \tau_{2}\right], \\
& \mathfrak{G}_{22}^{3}\left(\tau_{k}\right)=\underset{0 \leqq \lambda \leqq 2 \pi}{\mathcal{S}} \mathrm{E}\left[w_{k}=w_{k}\left(z_{1}, \tau_{2} e^{i \lambda}\right),\left|z_{1}\right| \leqq \tau_{1}\right],
\end{aligned}
$$

and their intersection $\mathfrak{F}_{2}^{2}\left(\tau_{k}\right)=\mathfrak{C}_{21}^{3}\left(\tau_{k}\right) \cdot \mathfrak{C}_{22}^{3}\left(\tau_{k}\right)$ is the distinguished boundary surface of $\mathfrak{B}_{2}\left(\tau_{k}\right)$.

We shall find it convenient to define a real (uniformly) continuous function $G_{2}\left(w_{1}, w_{2} ; \tau_{k}\right)$ in $\mathfrak{B}_{2}\left(\mathrm{P}_{k}\right)$ which is positive in $\mathfrak{B}_{2}\left(\tau_{k}\right)$, nonpositive in $\mathfrak{B}_{2}\left(\mathrm{P}_{k}\right)-\mathfrak{B}_{2}\left(\tau_{k}\right)$, and, therefore, zero on the boundary of $\mathfrak{B}_{2}\left(\tau_{k}\right)$. The existence of such functions follows from the fact that $\mathfrak{B}_{2}\left(\tau_{k}\right)$ is an image of a bicylinder $\mathfrak{B}_{1}\left(\tau_{k}\right)$; we can define an analogous function $G_{1}\left(z_{1}, z_{2} ; \tau_{k}\right)$ in $\mathfrak{B}_{1}\left(\mathrm{P}_{k}\right)$, and then $G_{1}\left[z_{1}\left(w_{1}, w_{2}\right), z_{2}\left(w_{1}, w_{2}\right) ; \tau_{k}\right]$ is a function possessing the indicated properties. However, given $\mathfrak{B}_{2}\left(\tau_{k}\right), G_{2}\left(w_{1}, w_{2} ; \tau_{k}\right)$ can be constructed without using the PT $\mathbf{w}^{-1}$.

7.4. We now discuss the surface

$$
\Im_{2}^{2}\left(\psi_{1}, \psi_{2}\right)=\mathrm{E}\left[\arg w_{k}=\psi_{k}=\text { const., }\left(w_{1}, w_{2}\right) \in \mathfrak{B}_{2}\left(\mathbf{P}_{k}\right)\right] .
$$

${ }^{(20)}$ The double subscripts are written without a comma; for example $\mathfrak{b}_{21}^{3}$ for $\mathfrak{b}_{2.1}^{3}$, the first, number, 1 or 2 , indicating the space $\left(z_{1} z_{2}\right)$ or $\left(w_{1} w_{2}\right)$. See $\S 2$. 
LEMMA 2. There exists a set( $\left.{ }^{21}\right) \mathfrak{S}^{2}, \mathrm{~m}\left(\mathfrak{S}^{2}\right)=4 \pi^{2}$, lying in the square $\mathrm{E}\left[0 \leqq \psi_{k}<2 \pi\right]$ such that, if $\left(\psi_{1}, \psi_{2}\right) \in \mathfrak{W}^{2}, \mathfrak{B}_{2}^{2} \cdot \Im_{2}^{2}\left(\psi_{1}, \psi_{2}\right)$ is composed of only finitely many points.

We remark that, since the number of branch points of the secord kind is finite, we can further suppose that $\mathfrak{S}_{2}^{2}\left(\psi_{1}, \psi_{2}\right)$ contains no such points if $\left(\psi_{1}, \psi_{2}\right) \in \mathfrak{S}^{2}$.

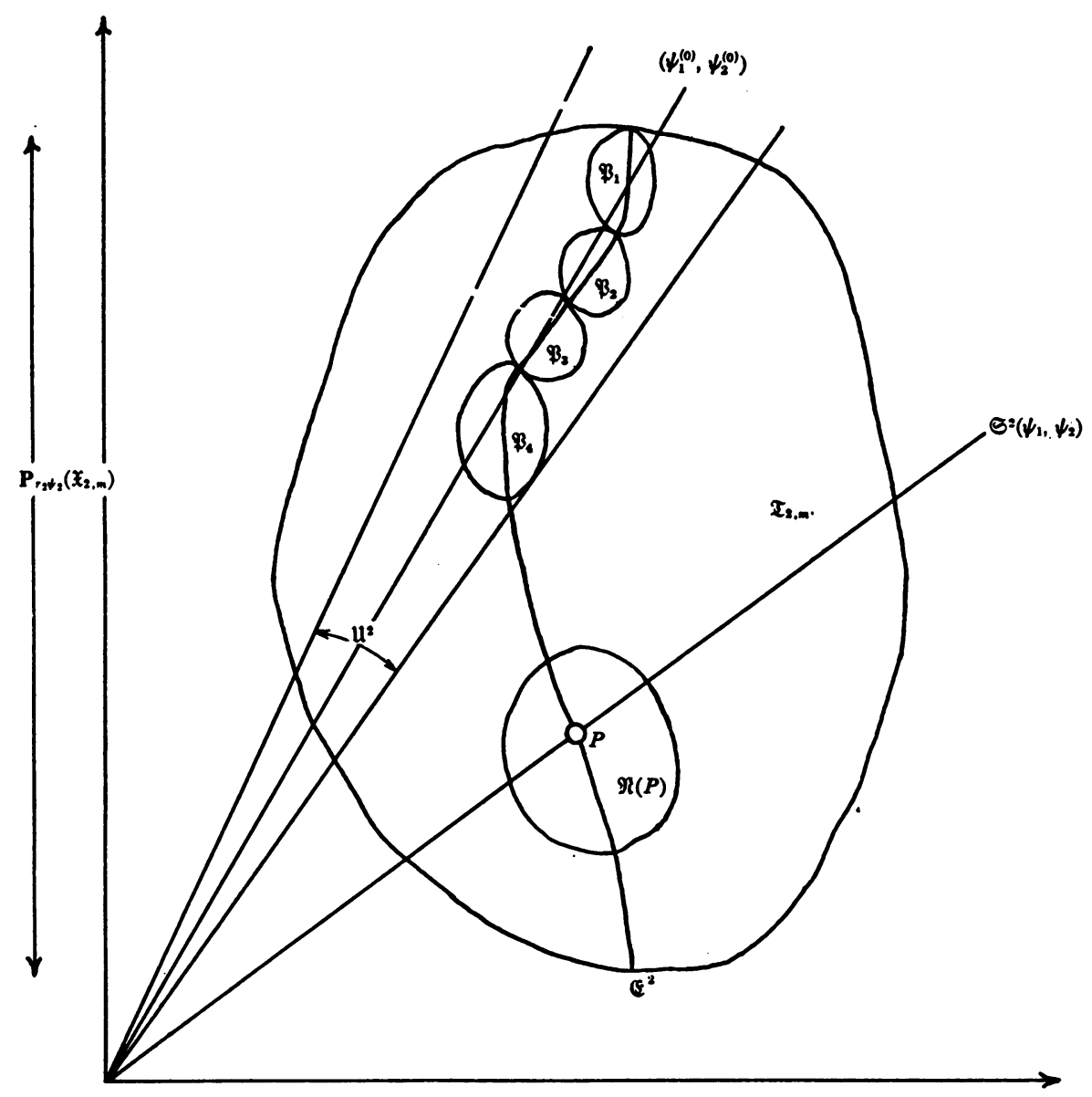

FIG. 2

Denoting branch points of the second kind by $\left(w_{1}^{(\nu)}, w_{2}^{(\nu)}\right), \nu=1,2, \cdots, p$, we can (by the Weierstrass preparation theorem and the Heine-Borel theorem) cover the domain $\mathfrak{B}_{2}\left(\mathbf{P}_{k}\right)-S_{\nu=1}^{p} E\left[\left|w_{1}-w_{1}^{(\nu)}\right|^{2}+\left|w_{2}-w_{2}^{(\nu)}\right|^{2}<\epsilon\right]$ with a

(12) $\mathrm{m}\left(\mathfrak{\Phi}^{2}\right)$ denotes the measure of $\mathfrak{W}^{2}$. 
finite number of domains $\mathfrak{I}_{2, m}$ in such a way that in each $\mathfrak{X}_{2, m}$ (the closure of $\left.\mathfrak{T}_{2, m}\right)$ the branch surface $\mathfrak{E}_{2}^{2}$ can be represented in the form $\left({ }^{22}\right)$

$$
w_{1}=H\left(w_{2}\right) \text {, }
$$

or

$$
w_{2}=H\left(w_{1}\right),
$$

where $H$ is an analytic function of one complex variable regular in the projection $\mathbf{P}_{u_{k}}\left[\mathfrak{X}_{2, m}\right]$ of $\mathfrak{X}_{2, m}$ on the $w_{k}$-plane (see $\S 2$ ).

Suppose that the first representation of $(7.4 .2)$ is valid for $\mathfrak{E}_{2}^{2} \cdot \mathfrak{T}_{2, m}$. Then (7.4.2) can be written in the form

$$
r_{1}=\left[H\left(r_{2} e^{i \psi_{2}}\right) \bar{H}\left(r_{2} e^{-i \psi_{2}}\right)\right]^{1 / 2}, \quad \psi_{1}=(1 / 2 i)\left[\lg H\left(r_{2} e^{i \psi_{2}}\right)-\lg H\left(r_{2} e^{-i \psi_{2}}\right)\right] .
$$

Since $H \neq 0, r_{1}$ and $\psi_{1}$ are one-valued and regular functions of $r_{2}$ and $\psi_{2}$. In the neighborhood $\mathfrak{N}(P)$ of any point $P$ of $\mathfrak{E}_{2}^{2} \cdot \mathfrak{T}_{2, m}$ in which

$$
D\left(r_{2}, \dot{\psi}_{2}\right)=\partial\left[\lg H\left(r_{2} e^{i \psi_{2}}\right)-\lg \bar{H}\left(r_{2} e^{-i \psi_{2}}\right)\right] / \partial r_{2} \neq 0,
$$

the surface $\mathfrak{E}_{2}^{2} \cdot \mathfrak{T}_{2, m}$ can also be written in the form

$$
r_{k}=t_{k}\left(\psi_{1}, \psi_{2}\right) \text {, }
$$

where the $t_{k}$ are one-valued functions of $\psi_{1}, \psi_{2}$, so that $\mathfrak{E}_{2}^{2} \cdot \mathfrak{S}_{2}^{2}\left(\psi_{1}, \psi_{2}\right) \cdot \mathfrak{R}(P)$ consists of only one point.

We shall determine for what values of $\left(\psi_{1}, \psi_{2}\right)$ the function $D\left(r_{2}, \psi_{2}\right)$ vanishes on $\mathfrak{F}_{2}^{2} \cdot \mathfrak{T}_{2, m}$. Since $D\left(r_{2}, \psi_{2}\right)$ is an analytic function of 2 real variables $r_{2}, \psi_{2}$, regular in $\mathbf{P}_{r_{2} \psi_{2}}\left(\mathfrak{X}_{2, m}\right)$, we can cover $\mathbf{P}_{r_{2} \psi_{2}}\left(\mathfrak{X}_{2, m}\right)$ with a finite number of neighborhoods $\mathfrak{U}_{m, \mu}^{2}$ in each of which the equation $D\left(r_{2}, \psi_{2}\right)=0$ can be expressed in the form

$$
\chi_{0}^{(m \mu)}\left(\psi_{2}\right) r_{2}^{p}+\chi_{1}^{(m \mu)}\left(\psi_{2}\right) r_{2}^{p-1}+\cdots+\chi_{p}^{(m \mu)}\left(\psi_{2}\right)=0 .
$$

Since $\chi_{0}^{(m \mu)}\left(\psi_{2}\right)$ has in every $\mathfrak{u}_{m, \mu}^{2}$ only a finite number of factors $\left(\psi_{2}-\psi_{2}^{(m \mu l)}\right)$, we can cover $\mathbf{P}_{r_{2} \psi_{2}}\left(\mathfrak{X}_{2, m}\right)$ (perhaps multiply) with a finite number of domains $\mathfrak{M}_{m \mu s}^{2}$ such that $D\left(r_{2}, \psi_{2}\right)=0$ can be written in $\mathfrak{M}_{m \mu s}^{2}$ in the form

$$
r_{2}=r_{2}^{(m \mu s)}\left(\psi_{2}\right)
$$

$$
\left(\psi_{2} \neq \psi_{2}^{(m \mu l)}\right),
$$

where the $r_{2}^{(m \mu s)}\left(\psi_{2}\right)$ are algebroid functions in $\mathbf{P}_{\psi_{2}}\left(\mathfrak{M}_{m \mu s}^{2}\right)$. Substituting from (7.4.7) into the second equation of (7.4.3), we see that $D\left(r_{2}, \psi_{2}\right)$ vanishes on $\mathfrak{E}_{2}^{2} \cdot \mathfrak{X}_{2, m}$ only for values of $\left(\psi_{1}, \psi_{2}\right)$ for which

$$
\psi_{1}=A^{(m \mu s)}\left(\psi_{2}\right) \text {, }
$$

or

$\left.{ }^{(22}\right)$ See Osgood [1, p. 113]. Figure 2 is a schematic diagram in which four-dimensional domains have been replaced by plane domains, and surfaces by curves. 


$$
\psi_{2}=\psi_{2}{ }^{(m \mu l)} \text {, }
$$

where the $A^{(m \mu s)}\left(\psi_{2}\right)$ are again algebroid functions in $\mathbf{P}_{\psi_{2}}\left(\mathfrak{M}_{m \mu s}^{2}\right)$.

In every $P_{\psi_{1} \psi_{2}}\left(\mathfrak{I}_{2, m}\right)$ each expression (7.4.8) defines a curve the two-dimensional measure of which is zero. Since the number of these curves is finite and since $m, \mu, s, l$ run through a finite set of integers, the measure of the set $\mathfrak{S}^{2}$ which is left after removing all these curves from $\mathrm{E}\left[0 \leqq \psi_{k}<2 \pi\right]$ will be $4 \pi^{2}$.

Consider now a neighborhood $\mathfrak{U}_{0}^{2}$ of a point $\left(\psi_{1}^{(0)}, \psi_{2}^{(0)}\right) \in \mathfrak{S}^{2}$. We can choose $\mathfrak{U}_{0}^{2}$ so small that $\mathfrak{Y}_{0}^{2} \subset \mathfrak{S}^{2}$ (where $\mathfrak{Y}_{0}^{2}$ is the closure of $\mathfrak{U}_{0}^{2}$ ). Since in every domain $\Omega_{m}=\mathfrak{X}_{2, m} \cdot \mathrm{E}\left[\left(\psi_{1}, \psi_{2}\right) \in \mathfrak{Y}_{0}^{2}\right]$, we have $D\left(r_{2}, \psi_{2}\right) \neq 0$ on $\Omega_{m} \cdot \mathfrak{E}_{2}^{2}$, we can cover $\Omega_{m}$ with a finite number of neighborhoods $\mathfrak{B}_{\mu}$ in each of which we have the representation (7.4.5) for $\mathfrak{P}_{\mu} \cdot \mathfrak{E}_{2}^{2} \cdot \mathfrak{S}_{2}^{2}\left(\psi_{1}, \psi_{2}\right)$. Since the $t_{k}\left(\psi_{1}, \psi_{2}\right)$ are one-valued functions, and since there is only a finite number of $m$ and $\mu, \mathcal{E}_{2}^{2} \cdot \mathfrak{S}_{2}^{2}\left(\psi_{1}, \psi_{2}\right)$ consists of a finite number of points for $\left(\psi_{1}, \psi_{2}\right) \in \mathfrak{U}_{0}^{2}$, and this proves Lemma 2 .

7.5. In this section we study the curves (for $\mathfrak{G}_{21}^{3}\left(\tau_{1}\right)$ see (7.3.1))

$$
\stackrel{1}{\mathfrak{C}_{21}}\left(\tau_{1}\right)=\mathfrak{S}_{2}^{2}\left(\psi_{1}, \psi_{2}\right) \cdot \mathfrak{C}_{21}^{3}\left(\tau_{1}\right),
$$

on which we suppose no branch points (that is, points of $\mathfrak{B}_{2}^{2}$ ) are situated, and where $\left(\psi_{1}, \psi_{2}\right)$ is a point of $\mathfrak{S}^{2}$. By (7.3.1) we may write (7.5.1) in the form

$$
\mathrm{c}_{21}^{1}\left(\tau_{1}\right)=\mathrm{E}\left[\tau_{1}=\left|z_{1}\left(r_{1} e^{i \psi_{1}}, r_{2} e^{i \psi_{2}}\right)\right|\right]
$$

If $r_{k}>0$, we call a point $\left(r_{1}, r_{2}\right)$ of the curve $c_{21}^{1}\left(\tau_{1}\right)$ for which (simultaneously)

$$
\partial\left(\left|z_{1}\right|\right) / \partial r_{1}=0, \quad \partial\left(\left|z_{1}\right|\right) / \partial r_{2}=0,
$$

a "node." A node is either an isolated point or a point at which (finitely) many different branches of the curve meet. If $\left(r_{1}^{0}, r_{2}^{0}\right)$ is neither a node nor a point of $\mathfrak{B}_{2}^{2}$, then, by a well known theorem of implicit funçtion-theory, we can represent $\mathfrak{c}_{21}^{1}\left(\tau_{1}\right)$ in a neighborhood of $\left(r_{1}^{0}, r_{2}^{0}\right)$ in one of the two following forms:

$$
r_{1}=a\left(r_{2}\right), \quad r_{2}=a\left(r_{1}\right),
$$

where $a$ is in both cases an analytic function of one real variable.

It follows, in particular, that if the curve $\mathfrak{c}_{21}^{1}\left(\tau_{1}\right), \mathfrak{c}_{21}^{1}\left(\tau_{1}\right) \cdot \mathfrak{B}_{2}^{2}=0$, ends in the interior of $\mathfrak{S}_{2}^{2}\left(\psi_{1}, \psi_{2}\right)$, it must end at a node. A piece of $\mathfrak{c}_{21}^{1}\left(\tau_{1}\right)$ which contains no node but which connects two nodes, two boundary points of $\mathfrak{S}_{2}^{2}\left(\psi_{1}, \psi_{2}\right)$, or a boundary point and a node, we call a branch of $c_{21}^{1}\left(\tau_{1}\right)$. Each branch is a regular curve $\left({ }^{23}\right)$.

Since the number of nodes is finite, and since finitely many branches meet

${ }^{(23)}$ That is to say, a curve whose equation is differentiable infinitely often. In particular, a branch has a tangent at every point including the end points (since at a node we have the representation (7.5.4), where $a\left(r_{k}\right)$ is an algebroid function). 
in a node, we see that there are only a finite number of branches of which at least one end point is a node.

The neighborhood on $\mathfrak{S}_{2}^{2}\left(\psi_{1}, \psi_{2}\right)$ of any interior point of a branch may consist of three kinds of points: (i) points corresponding to $\left|z_{1}\right|=\tau_{1}$, that is, points of $\mathfrak{c}_{21}^{1}\left(\tau_{1}\right)$; (ii) points corresponding to $\left|z_{1}\right|<\tau_{1}$; and (iii) points corresponding to $\left|z_{1}\right|>\tau_{1}$. We call points of the second kind $L\left(\tau_{1}\right)$-points, points of the third kind $M\left(\tau_{1}\right)$-points. We then say that a branch is of the first kind if $L$-points $\left({ }^{24}\right)$ lie on one side of it, $M$-points on the other; of the second (third) kind if $L$-points ( $M$-points) lie on both sides of it.

7.6. By means of the function $G_{2}\left(w_{1}, w_{2} ; 1\right)$ introduced above $(\$ 7.3)$, we divide all points of $\mathfrak{S}_{2}^{2}\left(\psi_{1}, \psi_{2}\right)$ into three categories; namely, $i$-points, $e$-points and $a$-points, at which $G_{2}\left(w_{1}, w_{2} ; 1\right)$ is positive, zero, or negative, respectively.

If $\left(\psi_{1}, \psi_{2}\right) \in \mathfrak{S}^{2}$ (see $\left.\$ 7.4\right)$, the totality of $i$-points of $\mathfrak{S}_{2}^{2}\left(\psi_{1}, \psi_{2}\right)$ forms a set which lies on a Riemann surface. By Lemma 2 there are at most finitely many branch points in this set, and each of them may be uniformized by (7.1.3). This set will consist in general of many disconnected components. Since (see §6) the mapping is schlicht in

$$
\mathfrak{D}_{1} \cdot\left[\left|z_{1}\right| \leqq \epsilon\right]+\mathfrak{D}_{1} \cdot\left[\left|z_{2}\right| \leqq \epsilon\right],
$$

and is the identical transformation $w_{k}=z_{k}$ on $\left|z_{1}\right|=0$ and $\left|z_{2}\right|=0$, there is one and only one component which contains the lines

$$
\stackrel{\mathfrak{a}_{21}}{t_{1}}=\mathrm{E}\left[0 \leqq r_{1} \leqq 1, r_{2}=0\right], \mathfrak{a}_{22}^{t_{1}}=\mathrm{E}\left[r_{1}=0,0 \leqq r_{2} \leqq 1\right]
$$

as part of its boundary. We denote this component by $\Im_{2}^{\dagger 2}\left(\psi_{1}, \psi_{2}\right)$. The boundary of $\mathfrak{S}_{2}^{\dagger 2}\left(\psi_{1}, \psi_{2}\right)$ consists of $\mathfrak{a}_{21}^{\dagger 1}$ and $\mathfrak{a}_{22}^{\dagger 1}$, and of pieces of $\mathfrak{c}_{21}^{1}(1)$ and $\mathfrak{c}_{22}^{1}(1)$ which we shall denote by $c_{21}^{\dagger 1}, c_{22}^{\dagger 1}$, respectively (see Figure 3 ). We shall require the following lemma.

Lemma 3. If $\left(\psi_{1}, \psi_{2}\right) \in \mathfrak{S}^{2}$, the projection of the surface

$$
\Im_{1}^{\dagger 2}\left(\psi_{1}, \psi_{2}\right)=\mathrm{w}^{-1}\left[\Im_{2}^{\dagger 2}\left(\psi_{1}, \psi_{2}\right)\right] \subset \mathrm{E}\left[\arg w_{k}\left(\rho_{1} e^{i \theta_{1}}, \rho_{2} e^{i \theta_{2}}\right)=\psi_{k}=\text { const. }\right]
$$

on the $\rho_{1} \rho_{2}$-plane [i.e., the totality of the $\left(\rho_{1}, \rho_{2}\right)$ coordinates of points of $\Im_{1}^{\dagger 2}\left(\psi_{1}, \psi_{2}\right)$ ] covers a set

$$
\Im^{2} \subset \mathrm{E}\left[0<\rho_{\mathrm{k}}<1\right]
$$

where $\mathrm{m}\left(\Im^{2}\right)=1$.

In fact, suppose that $0<\lambda<1$, and that there are no points of $\mathfrak{B}_{2}^{2}$ on $\mathfrak{c}_{21}^{1}(\lambda)$. Since the mapping is schlicht in a neighborhood of the line $\left|z_{1}\right|=\lambda,\left|z_{2}\right|=0$, one and only one branch $\mathfrak{a}_{1}^{1}$ of $\mathfrak{c}_{21}^{1}(\lambda)$ begins at the point $K_{0}=\left[r_{1}=\lambda, r_{2}=0\right]$. We shall show that by adding to $\mathfrak{a}_{1}^{1}$ further suitably chosen branches of $\mathfrak{c}_{21}^{1}(\lambda)$, we obtain a connected curve which cuits $c_{22}^{1}(1)$.

(2) We shall omit the parameter $\tau_{1}$ in $L\left(\tau_{1}\right)$ and $M\left(\tau_{1}\right)$ hereafter. 
Beginning at $K_{0}$ we travel outward along $\mathfrak{a}_{1}^{1}$ keeping $L$-points to the left until we reach a point of $\mathfrak{a}_{1}^{1}$ corresponding to

$$
\left[\left|z_{1}\left(w_{1}, w_{2}\right)\right|=\lambda,\left|z_{2}\left(w_{1}, w_{2}\right)\right|=1\right],
$$

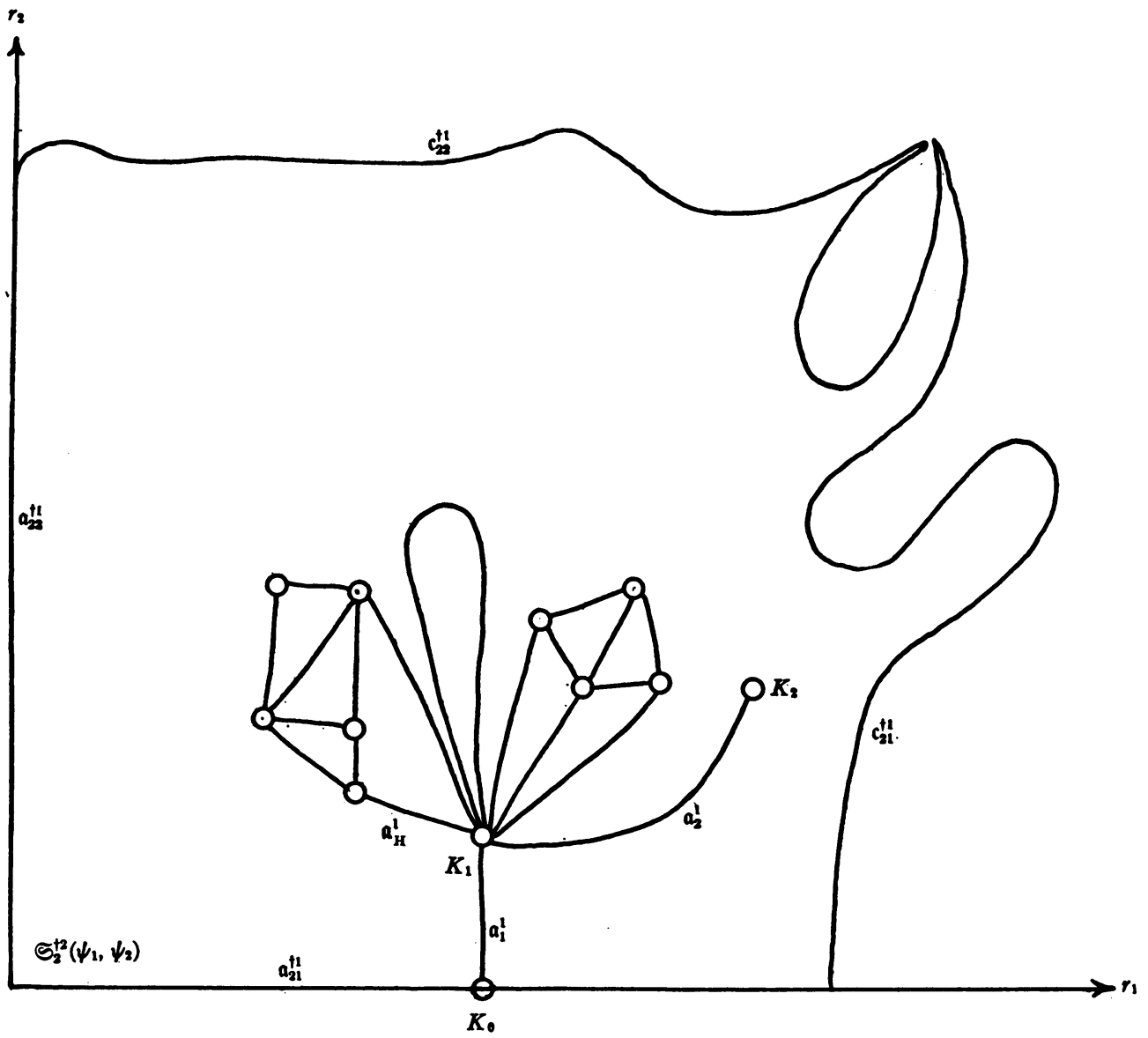

Fig. 3

or a node $K_{1}$ for which $\left.{ }^{25}\right)$

$$
\left|z_{1}\right|=\lambda,\left|z_{2}\right|<1 .
$$

In the latter case we start from the $L$-edge of $a_{1}^{1}$ and turn clockwise round a sufficiently small circle $\left({ }^{26}\right)$ with center $K_{1}$ until we meet a new branch $\mathfrak{a}_{\mathrm{H}}^{1}$ of the first kind. $a_{\mathrm{H}}^{1}$ exists; otherwise we should arrive at the end of $\mathfrak{a}_{1}^{1}$ where

(25) Hereafter, for simplicity, we shall write $\left|z_{1}\right|=\lambda,\left|z_{2}\right|=1$, and omit the argument $\left(w_{1}, w_{2}\right)$.

(20) The radius of the circle depends, of course, on the node. It is small enough so that it intersects branches with one end point at $K_{1}$ once and only once, branches with two end points at $K_{1}$ only twice, and intersects no branch which does not have an end point at $K_{1}$. 
$M$-points are situated. This is impossible since we cannot connect a point $L$ with a point $M$ without meeting a branch of the first kind( $\left.{ }^{27}\right)$.

We now travel along $\mathfrak{a}_{\mathrm{H}}^{1}$ with $L$-points to the left until we meet a point corresponding to $\left[\left|z_{1}\right|=\lambda,\left|z_{2}\right|=1\right]$, or a new node at which $\left|z_{1}\right|=\lambda,\left|z_{2}\right|<1$, and repeat the same process. It may happen that after running over a set of branches $\left(\mathfrak{a}_{\mathrm{H}}^{1}, \mathfrak{a}_{\mathrm{H}+1}^{1}, \cdots, \mathfrak{a}_{\mathrm{H}+\nu}^{1}\right), \nu \geqq 0$, we arrive back at the node $K_{1}$ from which we started without leaving the domain $E\left[\left|z_{1}\right|<1,\left|z_{2}\right|<1\right]$. If a set of branches chosen in this way has the property that only the first and the

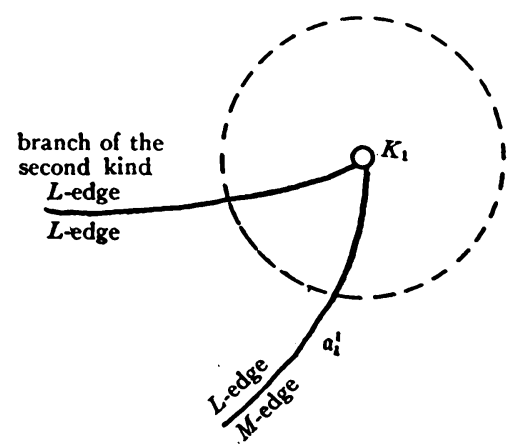

Fig. 4

last have end points at $K_{1}\left({ }^{28}\right)$, we call it a chain $\mathfrak{u}_{1}^{1}\left(K_{1}\right)$ hung from $K_{1}$. Since in turning clockwise round $K_{1}$ from $\mathfrak{a}_{1}^{1}$ to $\mathfrak{a}_{\mathrm{H}}^{1}$ we meet no branch of the first kind, we conclude that $a_{\mathrm{H}+\nu}^{1}$ must lie to the right of $\mathfrak{a}_{\mathrm{H}}^{1}$. The chain $\mathfrak{u}_{1}^{1}\left(K_{1}\right)$ has an important property: It is possible to draw a curve from the $L$-side of $\mathfrak{u}_{1}^{1}\left(K_{1}\right)$ which begins and ends at $K_{1}$, but which lies (apart from its end points and perhaps points lying on branches of the second kind) completely in an $L$-domain, and which, therefore, cuts $\left|z_{1}\right|=\lambda$ (if at all) only in branches of the second kind. This follows from the fact that in forming the chain we turn round each node $K_{p}$ in the clockwise sense until we meet a branch of the first kind.

After traveling over the branch $\mathfrak{a}_{\mathrm{H}+\nu}^{1}$ which leads to $K_{1}$, we move clockwise round a sufficiently small circle until we again meet a branch $\mathfrak{a}_{\mathfrak{l}}^{1}$, say, of the first kind, then continue along $\mathfrak{a}_{l}^{1}$, and so on. But we may now run over a second chain $\mathfrak{u}_{2}^{1}\left(K_{1}\right)$ which leads us over finitely many branches (situated in $\left.\mathrm{E}\left[\left|z_{1}\right|<1,\left|z_{2}\right|<1\right]\right)$ and then back to $K_{1}$ again. However, the chains $\mathfrak{u}_{1}^{1}\left(K_{1}\right)$ and $\mathfrak{u}_{2}^{1}\left(K_{1}\right)$ have no common node except $K_{1}$. For, in the neighborhood of $K_{1}$, $\mathfrak{a}_{l}^{1}$ lies on the $L$-side of $\mathfrak{u}_{1}^{1}\left(K_{1}\right)$. If, therefore, we move from $K_{1}$ along $\mathfrak{a}_{l}^{1}$, we

${ }^{(27)}$ For we must meet a branch. If the branch were of the second or the third kind we could connect an $L$-with an $M$-point without passing through a point for which $\left|z_{1}\right|=\lambda$.

${ }^{(28)}$ If the chain contains but one branch $\mathfrak{a}_{\mathbf{H}}^{1}$, we make a distinction between its two ends. 
must meet a branch $\mathfrak{a}_{l+p}^{1}$ which lies on the $L$-side of $\mathfrak{u}_{1}^{1}\left(K_{1}\right)$ and ends in a node $K_{n}$ at which two branches of the first chain $\mathfrak{a}_{\mathbf{H}+\mu}^{1}$ and $\mathfrak{a}_{\mathbf{H}+\mu+1}^{1}$ also end (see Figure 5). This is impossible; otherwise in turning clockwise round $K_{n}$, after having travelled $\mathfrak{a}_{\mathrm{H}+\mu}^{1}$, we should have met $\mathfrak{a}_{l+p}^{1}$, and not $\mathfrak{a}_{\mathrm{H}+\mu+1}^{1}$.

Continuing the process, we may travel over a set $\mathcal{C}\left(K_{1}\right)$ of chains $\mathfrak{u}_{1}^{1}\left(K_{1}\right)$, $\mathfrak{u}_{2}^{1}\left(K_{1}\right), \cdots$, hung from $K_{1}$; but no two chains of $\mathcal{C}$ have a common node other than $K_{1}$. Since in turning from $\mathfrak{a}_{1}^{1}$ clockwise round $K_{1}$ through $360^{\circ}$ we meet the initial before we meet the final branch of a chain, and since the number of branches attached to a node is finite, we see that the number

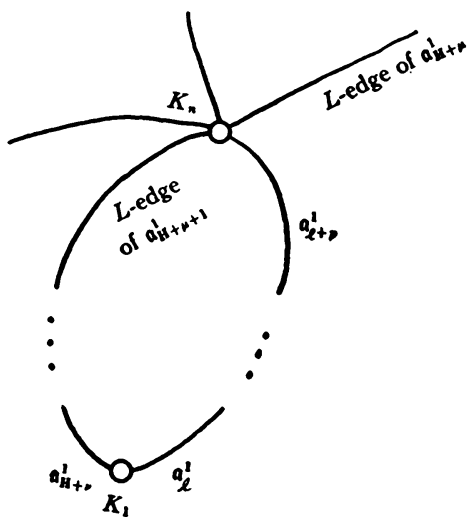

FIG. 5

of chains in $\mathcal{C}\left(K_{1}\right)$ is finite. By traveling round all chains hung from $K_{1}$ we must therefore find a branch, $\mathfrak{a}_{2}^{1}$, say, which will take us away from $K_{1}$ never to return; otherwise we could connect the $L$-edge of the last chain with the $M$-edge of $\mathfrak{a}_{1}^{1}$ without passing through a branch of the first kind, and this is impossible. $\mathfrak{a}_{2}^{1}$ leads us to a node $K_{2}$. At $K_{2}$ we proceed in the same manner as at $K_{1}$ with $\mathfrak{a}_{1}^{1}$ replaced by $\mathfrak{a}_{2}^{1}$. We cannot travel along $\mathfrak{a}_{2}^{1}$ a second time without passing through $K_{1}$ again, and this is again impossible. The continuation of our process, therefore, always leads us to new nodes, and since the number of nodes is finite, we must reach a branch on which a point corresponding to $\left|z_{1}\right|=\lambda,\left|z_{2}\right|=1$ lies. If not we should pass through all nodes and reach a branch $\mathfrak{a}_{s}^{1}$ of which one end point is a boundary point of $\mathfrak{S}_{2}^{2}\left(\psi_{1}, \psi_{2}\right)$. This end point corresponds neither to $\left|z_{1}\right|=0$ nor to $\left|z_{1}\right|=\mathrm{P}_{1}$; for our curve corresponds to $\left|z_{1}\right|=\lambda, 0<\lambda<1<\mathrm{P}_{1}$. Furthermore, it cannot be a point for which $\left|z_{2}\right|=0$; for we started from the point $K_{0}\left(r_{1}=\lambda, r_{2}=0\right)$ where, by hypothesis (see p. $147)$ there is only one branch, and we could, therefore, arrive at $K_{0}$ only by passing through $K_{1}$. It follows that the branch $\mathfrak{a}_{s}^{1}$ has one end at a point on $\left|z_{2}\right|=\mathrm{P}_{2}$, or at a node for which $\left|z_{2}\right|>1$. 
The system of branches $\left({ }^{29}\right)$

$$
\mathfrak{l}_{2}^{1}(\lambda)=\left(\mathfrak{a}_{1}^{1}, \mathfrak{a}_{2}^{1}, \cdots, \mathfrak{a}_{s-1}^{1}, \mathfrak{a}_{s, 0}^{1}\right)
$$

together forms a connected curve composed only of $i$-points.

Since, if $\epsilon$ is small enough, we can connect $\mathfrak{l}_{2}^{1}(\lambda)$ to a neighborhood of the point $r_{1}=0, r_{2}=0$, by means of the curve

$$
\mathrm{E}\left[\left|w_{2}\right|=\epsilon, \epsilon \leqq\left|w_{1}\right| \leqq \lambda\right]
$$

we see that $\mathfrak{l}_{2}^{1}(\lambda)$ lies in $\mathfrak{S}_{2}^{\dagger 2}\left(\psi_{1}, \psi_{2}\right)$. The curve $\mathfrak{l}_{1}^{1}(\lambda)=w^{-1}\left[\mathfrak{l}_{2}^{1}(\lambda)\right]$ is by Lemma 1 a connected curve with end points at $\left[\left|z_{1}\right|=\lambda,\left|z_{2}\right|=0\right]$ and $\left[\left|z_{1}\right|=\lambda,\left|z_{2}\right|=1\right]$, and its projection on the $\rho_{1} \rho_{2}$-plane is therefore $a$ fortiori a connected curve with end points at $\left[\rho_{1}=\lambda, \rho_{2}=0\right],\left[\rho_{1}=\lambda, \rho_{2}=1\right]$. Since $\rho_{1}=\lambda$ on the curve (by definition), the projection of $\mathfrak{l}_{1}^{1}(\lambda)$ is a straight line $E\left[\rho_{1}=\lambda, 0<\rho_{2}<1\right]$.

Since, by Lemma $2, \mathfrak{S}_{2}^{2}\left(\psi_{1}, \psi_{2}\right)$ contains only a finite number of points $\left(w_{1}^{(\mathbf{H})}, w_{2}^{(\mathbf{H})}\right)$ of $\mathfrak{B}_{2}^{2}$, there exists only a finite number of $\lambda^{(\mathbf{H})}=\left|z_{1}\left(w_{1}^{(\mathbf{H})}, w_{2}^{(\mathbf{H})}\right)\right|$ for which

$$
\left.\mathfrak{c}_{2}^{1} \lambda^{(\mathbf{H})}\right)=\mathrm{E}\left[\lambda^{(\mathbf{H})}=\left|z_{1}\left(w_{1}, w_{2}\right)\right|, \arg w_{k}=\psi_{k}\right]
$$

contains a point $\left(w_{1}^{(\mathrm{H})}, w_{2}^{(\mathrm{H})}\right)$ of $\mathfrak{B}_{2}^{2}$. For any other value of $\lambda$, and hence for almost all $\lambda$, the above argument is valid. Since for any $\lambda$ except $\lambda=\lambda(\boldsymbol{H}), \mathfrak{l}_{1}^{1}(\lambda)$ is contained in $S_{1}^{\dagger 2}\left(\psi_{1}, \psi_{2}\right)$, the projection of $\mathfrak{S}_{1}^{\dagger 2}\left(\psi_{1}, \psi_{2}\right)$ covers $\mathrm{E}\left(0<\rho_{k}<1\right)$ with exception of a null set. This completes the proof of Lemma 3.

7.7. In $\S 4$ we defined $\mathfrak{S}_{2}^{\dagger 2}\left(\psi_{1}, \psi_{2}\right),\left(\psi_{1}, \psi_{2}\right) \in \mathfrak{S}^{2}$, as that connected component of the set of $i$-points of $\Im_{2}^{2}\left(\psi_{1}, \psi_{2}\right)$ the boundary of which contains the point $w(0,0)$. We now define

$$
\mathfrak{B}_{2}^{\dagger}=\underset{\left(\psi_{1}, \psi_{2}\right) \in \mathfrak{W}^{2}}{\mathcal{S}_{2}^{\dagger_{2}}\left(\psi_{1}, \psi_{2}\right)}
$$

We remark, however, that our theorem is true for any domain

$$
\mathfrak{B}_{2}^{\dagger \dagger}=\underset{\left(\psi_{1}, \psi_{2}\right) \in \mathfrak{W}^{2}}{\mathcal{S}_{2}^{\dagger \dagger 2}\left(\psi_{1}, \psi_{2}\right)}
$$

which has the property that the projection of the surface

$$
\mathfrak{S}_{1}^{\dagger \dagger 2}\left(\psi_{1}, \psi_{2}\right)=\mathrm{w}^{-1}\left[\Im_{2}^{\dagger \dagger 2}\left(\psi_{1}, \psi_{2}\right)\right]
$$

fills the unit square $\mathrm{E}\left[0<\rho_{k}<1\right]$.

For example, from $\$ 7.6$ we see that we may take (in (7.7.2)) the surface

$$
\mathfrak{S}_{2}^{\dagger \dagger 2}\left(\psi_{2}, \psi_{2}\right)=\underset{0<\lambda<1}{S} \mathfrak{l}_{2}^{1}(\lambda)
$$

(29) $\mathfrak{a}_{0,0}^{1}$ here denotes only the portion of the last branch for which $\left|z_{2}\right|<1$. 
However, the resulting domain $\mathfrak{B}^{\text {t† }}$ has the disadvantage that it is defined by means of the PT $w$, whereas the domain $\mathfrak{B}^{\dagger}$ is obtained from $\mathfrak{B}_{2}$ by a geometrical operation definable without reference to $\mathrm{w}$.

\section{Proof. Analytical part}

8.1. We begin by restating the definition of $B$-area (see Bergman [2]). Let

$$
\begin{aligned}
\mathfrak{R}^{2}=\mathrm{E}\left[Z_{1}\left(u_{1}, u_{2}\right)\right. & =\chi_{1}\left(u_{1}, u_{2}\right)+i \chi_{2}\left(u_{1}, u_{2}\right), \\
Z_{2}\left(u_{1}, u_{2}\right) & \left.=\chi_{3}\left(u_{1}, u_{2}\right)+i \chi_{4}\left(u_{1}, u_{2}\right), 0 \leqq u_{k} \leqq 1\right],
\end{aligned}
$$

the $\chi_{j}\left(u_{1}, u_{2}\right)$ being real functions of two real variables which have continuous derivatives. Then $\mathrm{B}\left(\mathfrak{R}^{2}\right)$, the $B$-area of $\mathfrak{R}^{2}$, is defined by

$$
\mathrm{B}\left(\mathfrak{R}^{2}\right)=\int_{0}^{1} \int_{0}^{1} \mathrm{~b}\left(Z_{1}, Z_{2}\right) d u_{1} d u_{2}, \quad \mathrm{~b}\left(Z_{1}, Z_{2}\right)=\left|\partial\left(Z_{1}, Z_{2}\right) / \partial\left(u_{1}, u_{2}\right)\right| ;
$$

whereas $A\left(\mathfrak{R}^{2}\right)$, the ordinary area of it, is defined by

$$
\int_{0}^{1} \int_{0}^{1} \mathrm{a}\left(Z_{1}, Z_{2}\right) d u_{1} d u_{2}
$$

where $\left({ }^{30}\right)$

$\mathrm{a}\left(Z_{1}, Z_{2}\right)=E G-F^{2}=\left|\begin{array}{ll}\sum_{j u_{1}}^{2} & \sum \chi_{j u_{1} \chi_{j u_{2}}}^{2} \\ \sum \chi_{j u_{1}} \chi_{j u_{2}} & \sum \chi_{j u_{2}}^{2}\end{array}\right|, \quad \sum=\sum_{j=1}^{4}, \chi_{j u_{k}}=\frac{\partial \chi_{j}}{\partial u_{k}}$.

We have, therefore,

$$
\left[\mathrm{b}\left(Z_{1}, Z_{2}\right)\right]^{2}=E G-F^{2}-T^{2},
$$

where

$$
\begin{aligned}
T= & -\chi_{1 u_{1}} \chi_{2 u_{2}}+\chi_{2 u_{1}} \chi_{1 u_{2}}-\chi_{3 u_{1}} \chi_{4 u_{2}}+\chi_{4 u_{1}} \chi_{3 u_{2}} \\
= & \frac{1}{4 i} \sum_{k=1}^{2}\left[-\left(\frac{\partial Z_{k}}{\partial u_{1}}+\frac{\partial \bar{Z}_{k}}{\partial u_{1}}\right)\left(\frac{\partial Z_{k}}{\partial u_{2}}-\frac{\partial \bar{Z}_{k}}{\partial u_{2}}\right)\right. \\
& \left.+\left(\frac{\partial Z_{k}}{\partial u_{2}}+\frac{\partial \bar{Z}_{k}}{\partial u_{2}}\right)\left(\frac{\partial Z_{k}}{\partial u_{1}}-\frac{\partial \bar{Z}_{k}}{\partial u_{1}}\right)\right] \\
= & \operatorname{Im}\left[\sum_{k=1}^{2} \frac{\partial Z_{k}}{\partial u_{1}} \frac{\partial \bar{Z}_{k}}{\partial u_{2}}\right]
\end{aligned}
$$

and hence $\left({ }^{31}\right)$

(30) $E, F$ and $G$ are the coefficients of the fundamental form for the line-element.

(3) See Bergman $[2$, p. 476]. 


$$
\begin{aligned}
\mathrm{b}\left(Z_{1}, Z_{2}\right) & =\left(E G-F^{2}-T^{2}\right)^{1 / 2} \geqq \mathrm{a}\left(Z_{1}, Z_{2}\right)-|T| \\
& \geqq \mathrm{a}\left(Z_{1}, Z_{2}\right)-\sum_{k=1}^{2}\left|\frac{\partial Z_{k}}{\partial u_{1}} \frac{\partial Z_{k}}{\partial u_{2}}\right| .
\end{aligned}
$$

8.2. We recall that $w_{k}=r_{k} e^{i \psi_{k}}$; write $\mathfrak{S}_{2, \lambda}^{\dagger 2}\left(\psi_{1}, \psi_{2}\right)$ for the portion of the surface $\Im_{2}^{\dagger 2}\left(\psi_{1}, \psi_{2}\right)$ for which $2\left|w_{1} w_{2}\right|>\lambda$, and write $L_{1}, L_{2}$ for the two PT's $Z_{k}=\lg z_{k}, W_{k}=\lg w_{k}$, respectively. We then define

$$
\begin{aligned}
B_{1, \lambda}\left(\psi_{1}, \psi_{2}\right) & =\mathrm{B}\left\{\mathrm{L}_{1} \mathrm{~W}^{-1}\left[\Im_{2, \lambda}^{\dagger 2}\left(\psi_{1}, \psi_{2}\right)\right]\right\} \\
& =\iint_{\mathcal{S}_{2, \lambda}^{\dagger 2}\left(\psi_{1}, \psi_{2}\right)}\left|\frac{\partial\left(Z_{1}, Z_{2}\right)}{\partial\left(\lg w_{1}, \lg u_{2}\right)}\right| d\left(\lg w_{1}\right) d\left(\lg w_{2}\right) \\
& =\iint_{\mathcal{S}_{2, \lambda}^{\dagger 2}\left(\psi_{1}, \psi_{2}\right)}\left|\frac{\partial\left(Z_{1}, Z_{2}\right)}{\partial\left(r_{1}, r_{2}\right)}\right| d r_{1} d r_{2} .
\end{aligned}
$$

8.3. We prove the following lemma.

Lemma 4. Suppose that hypothesis (5.1.2) of the theorem is satisfied. Then, if $0<\lambda<2$,

$$
\begin{gathered}
\int_{-\pi}^{\pi} \int_{-\pi}^{\pi} B_{1, \lambda}\left(\psi_{1}, \psi_{2}\right) d \psi_{1} d \psi_{2} \geqq 2 \pi^{2} \lg ^{2} \lambda+4 \pi^{2} \lg 2 \cdot \lg (1 / \lambda)-\alpha_{2} \\
-O[\lambda \lg (1 / \lambda)] .
\end{gathered}
$$

Taking $u_{k}=r_{k}$, we have from (8.1.5) and (8.2.1) that

$$
B_{1, \lambda}\left(\psi_{1}, \psi_{2}\right) \geqq \iint_{\mathcal{S}_{2, \lambda}^{\dagger 2}\left(\psi_{1}, \psi_{2}\right)} \mathrm{a}\left(Z_{1}, Z_{2}\right) d r_{1} d r_{2}
$$

$$
-\iint_{\Theta_{2}^{\dagger 2}\left(\psi_{1}, \psi_{2}\right)} \mathrm{e}\left(w_{1}, w_{2}\right) d r_{1} d r_{2}
$$

where $Z_{k}=\lg \left[z_{k}\left(r_{1} e^{i \psi_{1}}, r_{2} e^{i \psi_{2}}\right)\right]$, and

$$
\mathrm{e}\left(w_{1}, w_{2}\right)=\sum_{k=1}^{2}\left|z_{k}\right|^{-2}\left|\left(\partial z_{k} / \partial w_{1}\right) \cdot\left(\partial z_{k} / \partial w_{2}\right)\right| \text {. }
$$

Let

$$
\begin{aligned}
& \mathfrak{P}_{1, \lambda}^{2}=\mathbf{P}_{\mathbf{l g}_{\rho_{1}, 1 \mathrm{~g} \rho_{2}}}\left\{\mathbf{L}_{1} \mathbf{W}^{-1}\left[\Im_{2, \lambda}^{t_{2}}\left(\psi_{1}, \psi_{2}\right)\right]\right\} \\
& \mathfrak{T}_{1, \lambda}^{2}=\mathbf{E}\left[\lambda \leqq 2 \rho_{1} \rho_{2}<2, \rho_{k}<1\right]
\end{aligned}
$$

Since by (6.2.3)

$$
2\left|z_{1}\left(w_{1}, w_{2}\right) \cdot z_{2}\left(w_{1}, w_{2}\right)\right| \leqq \lambda+O\left(\lambda^{2}\right)
$$




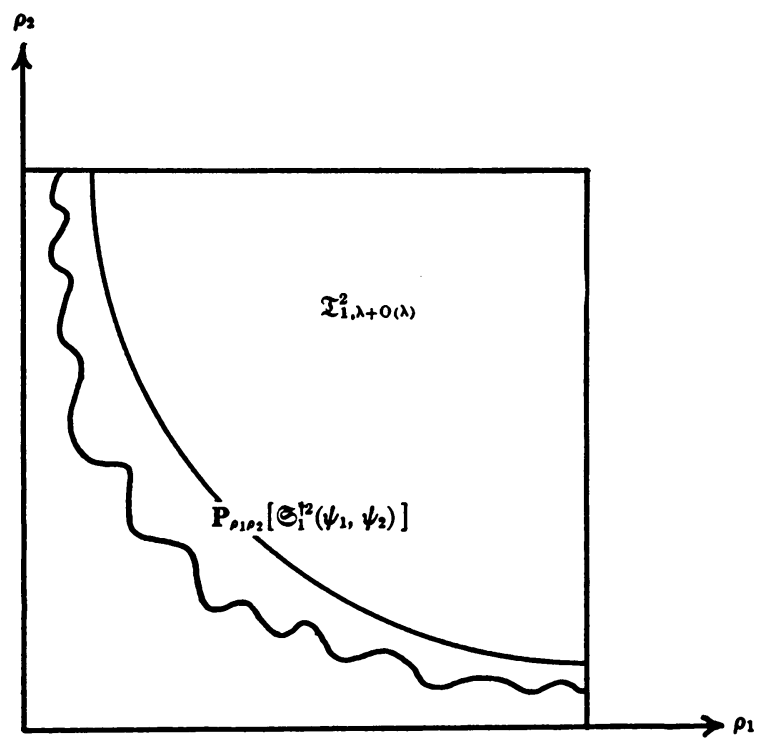

FIG. 6

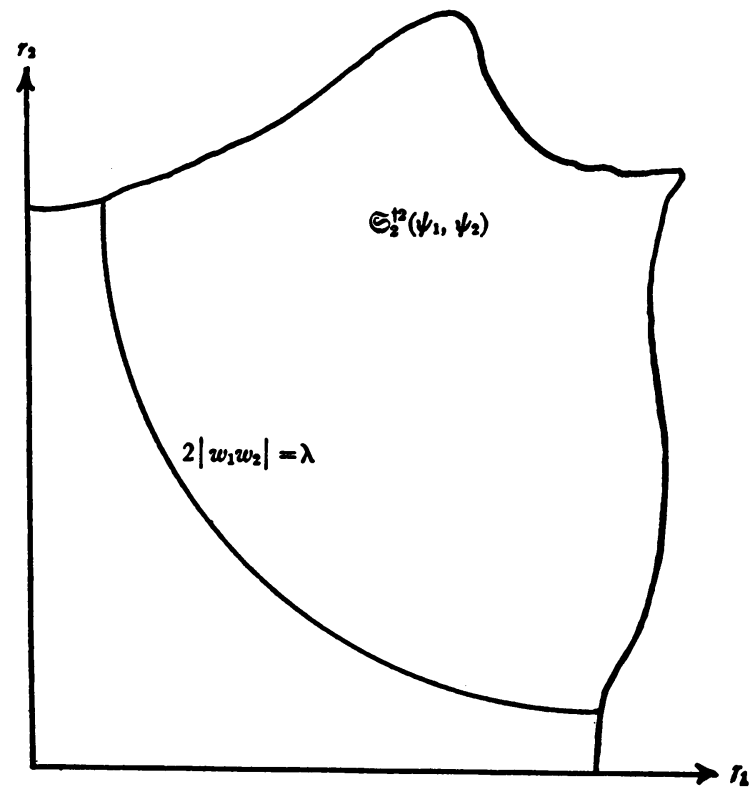

Fig. 7

for $2\left|w_{1} w_{2}\right|=\lambda$ we see that the boundary of $\mathfrak{B}_{1, \lambda}^{2}$ lies outside $L_{1}\left[\mathfrak{T}_{1, \lambda+o\left(\lambda^{2}\right)}^{2}\right]$ 
(see Figure 8). Suppose now that $\left(\psi_{1}, \psi_{2}\right) \in \mathfrak{S}^{2}, \mathrm{~m}\left(\mathfrak{Q}^{2}\right)=4 \pi^{2}$, where $\mathfrak{S}^{2}$ is the set in Lemma 3 . Then by Lemma 3 every point of $L_{1}\left[\mathfrak{T}_{1, \lambda+O\left(\lambda^{2}\right)}^{2}\right]$, with exception of a set of measure zero, is a point of $\mathfrak{P}_{1, \lambda}^{2}$; and so

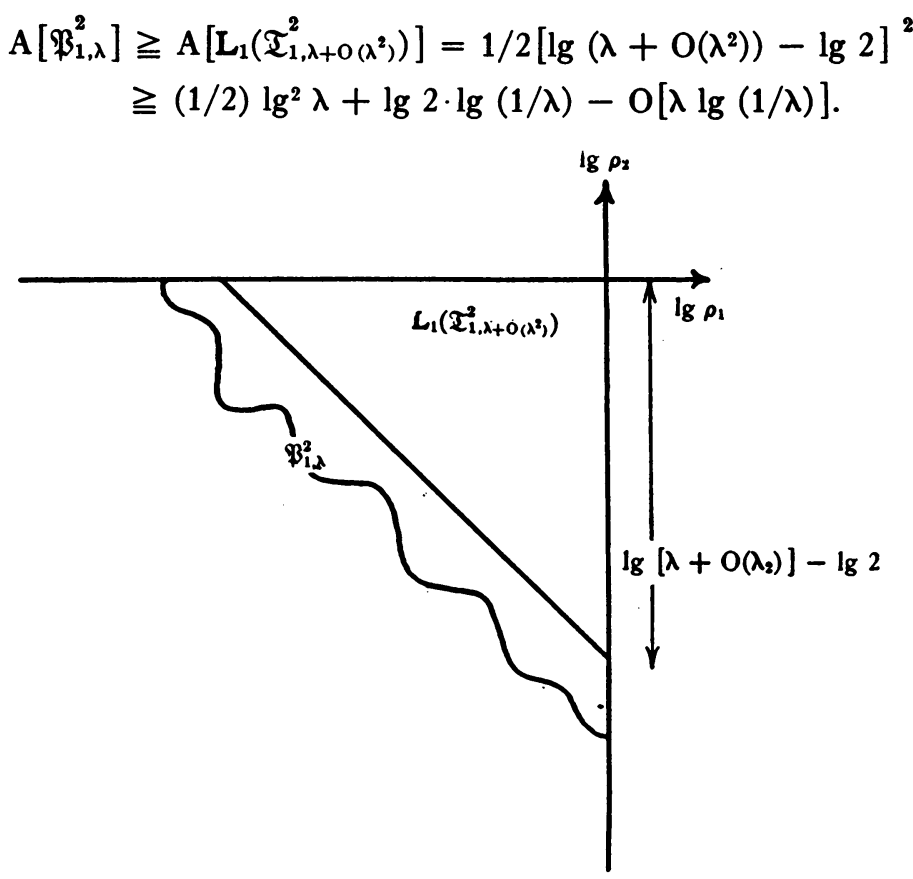

FIG. 8 .

Hence by (8.3.5) we have for $\left(\psi_{1}, \psi_{2}\right) \in \mathfrak{S}^{2}$, and therefore for almost âll $\left(\psi_{1}, \psi_{2}\right)$. that

$\iint_{(8.3 .6)} \int_{\mathscr{S}_{2, \lambda}^{\dagger_{2}}\left(\psi_{1}, \psi_{2},\right)} \mathrm{a}\left(Z_{1}, Z_{2}\right) d r_{1} d r_{2}=\mathrm{A}\left\{\mathrm{L}_{1} \mathrm{w}^{-1}\left[\Im_{2, \lambda}^{\dagger 2}\left(\psi_{1}, \psi_{2}\right)\right]\right\} \geqq \mathrm{A}\left(\mathfrak{B}_{1, \lambda}^{2}\right)$
$\geqq(1 / 2) \lg ^{2} \lambda+\lg 2 \cdot \lg (1 / \lambda)-\mathrm{O}[\lambda \lg (1 / \lambda)]$.

Finally,

$$
\begin{aligned}
\int_{-\pi}^{\pi} \int_{-\pi}^{\pi}\left[\iint_{\mathbb{S}_{2}^{\dagger_{2}}\left(\psi_{1}, \psi_{2}\right)} \mathrm{e}\left(w_{1}, w_{2}\right) d r_{1} d r_{2}\right] d \psi_{1} d \psi_{2} \\
\quad=\iiint \int_{\mathfrak{B}_{2}^{\dagger}}\left[\mathrm{e}\left(w_{1}, w_{2}\right) /\left|w_{1} w_{2}\right|\right] d \omega_{w} \\
\quad=\iiint \int_{\mathbb{W}^{-1}\left(\mathfrak{P}_{2}^{\dagger}\right) \leqq \alpha_{2}}\left[\sum_{k=1}^{2} \frac{1}{\left|z_{k}\right|^{2}}\left|\frac{\partial w_{1}}{\partial z_{3-k}} \cdot \frac{\partial w_{2}}{\partial z_{3-k}}\right|\right] \frac{d \omega_{z}}{\left|w_{1} w_{2}\right|} \\
\quad \leqq \alpha_{2} .
\end{aligned}
$$


by (5.1.2). Substituting from (8.3.6) into (8.3.2), integrating both sides of (8.3.2) with respect to $\psi_{k}$ over the square $-\pi \leqq \psi_{k}<\pi$, and using (8.3.7), we obtain (8.3.1).

8.4. By using the method of Bergman [4, pp. 147-149], we now obtain an inequality which is a generalization of a result in one variable (see Spencer [3]). Suppose that the PT

$$
\mathrm{t}: \zeta_{k}=\zeta_{k}\left(\sigma_{1}, \sigma_{2}\right)
$$

where $\sigma_{k}=\xi_{k}+i \eta_{k}$, transforms a domain $\mathfrak{G}_{2}$ into a domain $\mathfrak{G}_{1}$. Let $\mathfrak{Q}^{2}\left(\eta_{1}, \eta_{2}\right)$ be a set of points defined for each point $\left(\eta_{1}, \eta_{2}\right)$ of a set $\mathfrak{A}^{2}$, and contained in the intersection $\mathrm{B}_{2} \cdot\left(\eta_{k}=\right.$ const. $)$. Let

$$
B\left(\eta_{1}, \eta_{2}\right)=\iint_{\mathcal{Q}^{2}\left(\eta_{1}, \eta_{2}\right)}\left|\partial\left(\zeta_{1}, \zeta_{2}\right) / \partial\left(\sigma_{1}, \sigma_{2}\right)\right| d \xi_{1} d \xi_{2},
$$

$$
V_{2}=\mathrm{V}\left[\underset{\left(\eta_{1}, \eta_{2}\right) \in \mathfrak{H}^{2}}{S} \mathfrak{Q}^{2}\left(\eta_{1}, \eta_{2}\right)\right], \quad V_{1}=\mathrm{V}\left\{\mathrm{t}\left[\underset{\left(\eta_{1}, \eta_{2}\right) \in \mathfrak{x}^{2}}{S} \mathfrak{Q}^{2}\left(\eta_{1}, \eta_{2}\right)\right]\right\}
$$

We then have

Lemma 5.

$$
\left[\iint_{\mathfrak{Q}^{2}} B\left(\eta_{1}, \eta_{2}\right) d \eta_{1} d \eta_{2}\right]^{2} \leqq V_{1} V_{2}
$$

The proof is trivial. In fact, writing

$$
\mathrm{b}=\left|\partial\left(\zeta_{1}, \zeta_{2}\right) / \partial\left(\sigma_{1}, \sigma_{2}\right)\right|, \quad d \omega=d \xi_{1} d \xi_{2} d \eta_{1} d \eta_{2}, \quad \Im=\underset{\left(\eta_{1}, \eta_{2}\right) \in \mathfrak{R}^{2}}{\mathcal{Q}} \mathfrak{Q}^{2}\left(\eta_{1}, \eta_{2}\right),
$$

we have

$$
\begin{aligned}
{\left[\iint_{\mathfrak{x}^{2}} B\left(\eta_{1}, \eta_{2}\right)\right]^{2} } & =\left[\iiint \int_{\Im} \mathrm{b} d \omega\right]^{2} \\
& \leqq\left[\iiint \int_{\Im} \mathrm{b}^{2} d \omega\right] \cdot\left[\iiint \int_{\Im} d \omega\right]=V_{1} V_{2},
\end{aligned}
$$

by the inequality of Schwarz.

8.5. Let $\mathfrak{B}_{2, \lambda_{0}}^{\dagger}$ denote the portion of $\mathfrak{B}_{2}^{\dagger}$ for which $2\left|w_{1} w_{2}\right|>\lambda_{0}$. We transform the domain $L_{2}\left(\mathfrak{B}_{2, \lambda_{0}}^{\dagger}\right)$ by means of the PT $L_{1} w^{-1} L_{2}^{-1}$, and apply Lemma 5 with $\mathfrak{H}_{2}=\mathrm{L}_{2}\left(\mathfrak{B}_{2, \lambda_{0}}^{\dagger}\right), \mathfrak{G}_{1}=\mathrm{L}_{1} \mathbf{w}^{-1}\left(\mathfrak{B}_{2, \lambda_{0}}^{\dagger}\right), \eta_{k}=\psi_{k}, \xi_{k}=\lg r_{k}$, and

$$
\mathfrak{A}^{2}=\mathrm{E}\left[-\pi \leqq \psi_{k}<\pi\right] \text {. }
$$

We take

$$
\mathfrak{Q}^{2}\left(\eta_{1}, \eta_{2}\right)=\mathfrak{Q}^{2}\left(\psi_{1}, \psi_{2}\right)=\mathbf{L}_{2}\left[\Im_{2, \lambda_{0}}^{t_{2}}\left(\psi_{1}, \psi_{2}\right)\right] .
$$

Then by Lemma 4 


$$
\begin{aligned}
\iint_{\mathfrak{Q}^{2}} B\left(\eta_{1}, \eta_{2}\right) d \eta_{1} d \eta_{2}= & \int_{-\pi}^{\pi} \int_{-\pi}^{\pi} B_{1, \lambda_{0}}\left(\psi_{1}, \psi_{2}\right) d \psi_{1} d \psi_{2} \\
\geqq & 2 \pi^{2} \lg ^{2} \lambda_{0}+4 \pi^{2} \lg 2 \cdot \lg \left(1 / \lambda_{0}\right)-\alpha_{2} \\
& -\mathrm{O}\left[\lambda_{0} \lg \left(1 / \lambda_{0}\right)\right],
\end{aligned}
$$

if $0<\lambda_{0}<2$ (as we suppose). Hence by Lemma 5

$$
\begin{aligned}
2 \pi^{2} \lg ^{2} \lambda_{0}+4 \pi^{2} \lg 2 \cdot \lg \left(1 / \lambda_{0}\right)-\alpha_{2}- & O\left[\lambda_{0} \lg \left(1 / \lambda_{0}\right)\right] \\
& \leqq\left(V_{1} \cdot V_{2}\right)^{1 / 2} \leqq(1 / 2)\left(V_{1}+V_{2}\right),
\end{aligned}
$$

where

$$
V_{2}=\mathrm{V}\left[\mathrm{L}_{2}\left(\mathfrak{B}_{2, \lambda_{0}}^{\dagger}\right)\right], \quad V_{1}=\mathrm{V}\left[\mathrm{L}_{1} \mathrm{w}^{-1}\left(\mathfrak{B}_{2, \lambda_{0}}^{\dagger}\right)\right] .
$$

Let $\mathbf{L}$ denote the PT, $\mathbf{T}_{k}=\lg \tau_{k}$. The inequality (8.5.2) provides us with a lower bound in terms of $\lambda_{0}$ for the sum of $V\left[L_{2}\left(\mathfrak{B}_{2, \lambda_{0}}^{\dagger}\right)\right]$ and $V\left[L_{1} w^{-1}\left(\mathfrak{B}_{2, \lambda_{0}}^{\dagger}\right)\right]$. To obtain the statement (5.1.3) of the theorem - that is, to obtain a lower bound for $\mathrm{V}\left(\mathfrak{B}_{2, \lambda_{0}}^{\dagger}\right) \cdot \mathrm{V}\left[\mathrm{w}^{-1}\left(\mathfrak{B}_{2, \lambda_{0}}^{\dagger}\right)\right]$-we must rid (8.5.2) of the transformations $\mathbf{L}$. We note in this connection that the magnification by $\mathbf{L}$ of an element of volume at $\left(\lambda, \mu, \psi_{1}, \psi_{2}\right)$ is proportional to $\lambda^{-2}$. Therefore, to derive a lower bound for $V(\mathfrak{B})$ from a lower bound for fixed $V[L(\mathscr{B})]$, we must know something about the distribution of $\mathscr{B}$ with respect to $\lambda=0$. This information is supplied here by the "mean valency" hypothesis on $\mathfrak{B}_{2}^{\dagger}$.

8.6. Since the $w_{k}$ are regular in $\mathfrak{D}_{1}$, we have

$$
\mathrm{V}\left(\mathfrak{B}_{2, \lambda_{0}}^{\dagger}\right) \leqq \mathrm{V}\left(\mathfrak{B}_{2}^{\dagger}\right)<\infty .
$$

For brevity we now write

$$
G(\Lambda)=(1 / 2) \int_{0}^{\Lambda}|\lg (2 / \lambda)| d\left(\pi^{2} \lambda^{2}\right), E(\Lambda)=G(\Lambda)+\alpha_{1} \Lambda^{3} F(\Lambda),
$$

where $F$ is the function introduced in $\$ 4.5 .(G(\Lambda)$ is the volume of the portion of $\mathfrak{B}_{0}$ for which $2\left|w_{1} w_{2}\right|<\Lambda$.) Now we may plainly suppose (without loss of generality) that $E(\Lambda)$ is continuous. Then by (8.6.1) there exists a number $\Lambda_{2}, 0<\Lambda_{2}<\infty$, such that

$$
\mathrm{V}\left(\mathfrak{B}_{2}^{\dagger}\right)=E\left[\Lambda_{2}\right]
$$

We let

$$
\mathrm{n}^{\dagger}\left(r_{1}, r_{2}, \psi_{1}, \psi_{2}\right)=\mathrm{n}_{\mathfrak{B}_{2}^{\dagger}}\left(r_{1}, r_{2}, \psi_{1}, \psi_{2}\right)
$$

be the number of times $\mathfrak{B}_{2}^{\dagger}$ covers the point $\left(r_{1}, r_{2}, \psi_{1}, \psi_{2}\right), w_{k}=r_{k} e^{i \psi_{k}}$, and let

$$
\mathrm{n}_{1}^{\dagger}\left(\lambda, \mu, \psi_{1}, \psi_{2}\right)=\mathrm{n}^{\dagger}\left(r_{1}, r_{1}, \psi_{1}, \psi_{2}\right),
$$

where 


$$
2 r_{1} r_{1}=\lambda, \mu=r_{1}^{2}-r_{2}^{2}, d r_{1} d r_{2}=d \mu d \lambda / 4\left(\lambda^{2}+\mu^{2}\right)^{1 / 2} .
$$

Then

$$
\begin{aligned}
V_{2} & =\int_{-\pi}^{\pi} \int_{-\pi}^{\pi} \iint_{\mathrm{E}\left[2 r_{1} r_{2}>\lambda_{0}\right]} \mathrm{n}^{\dagger}\left(r_{1}, r_{2}, \psi_{1}, \psi_{2}\right) r_{1}^{-1} r_{2}^{-1} d r_{1} d r_{2} d \psi_{1} d \psi_{2} \\
& =\int_{-\pi}^{\pi} \int_{-\pi}^{\pi} \int_{\lambda_{0}}^{\infty} \int_{-\infty}^{\infty} \mathrm{n}_{1}^{\dagger}\left(\lambda, \mu, \psi_{1}, \psi_{2}\right) \cdot \frac{1}{4}\left(\lambda^{2}+\mu^{2}\right)^{-1 / 2} d \mu d\left(\lg \lambda^{2}\right) d \psi_{1} d \psi_{2} \\
& =\int_{\lambda_{0}}^{\infty} \mathrm{p}(\lambda) d\left(4 \pi^{2} \lg \lambda^{2}\right)
\end{aligned}
$$

by (4.3.1) and (4.3.2). Next, integrating by parts,

$$
\begin{aligned}
\int_{\lambda_{0}}^{\infty} \mathrm{p}(\lambda) d\left(\lg \lambda^{2}\right)= & \int_{\lambda_{0}}^{\infty} \mathrm{p}(\lambda) \cdot \lambda^{-2} d\left(\lambda^{2}\right)=\left[\Lambda^{-2} \int_{\lambda_{0}}^{\Lambda} \mathrm{p}(\lambda) d\left(\lambda^{2}\right)\right]_{\Lambda=\lambda_{0}}^{\Lambda=\infty} \\
& +2 \int_{\lambda_{0}}^{\infty}\left[\int_{\lambda_{0}}^{\Delta} \mathrm{p}(\lambda) d\left(\lambda^{2}\right)\right] \Lambda^{-3} d \Lambda \\
& =2 \int_{\lambda_{0}}^{\infty}\left[\int_{\lambda_{0}}^{\Delta} \mathrm{p}(\lambda) d\left(\lambda^{2}\right)\right] \Lambda^{-3} d \Lambda,
\end{aligned}
$$

and hence, substituting from (8.6.6) into (8.6.5), we have that

(8.6.7) $V_{2}=8\left\{\int_{\lambda_{0}}^{\Lambda_{2}}+\int_{\Delta_{2}}^{\infty}\right\}\left[\int_{\lambda_{0}}^{\Delta} \mathrm{p}(\lambda) d\left(\pi^{2} \lambda^{2}\right)\right] \Lambda^{-3} d \Lambda=I_{1}+I_{2}$,

say. Now

$$
\int_{0}^{\Lambda} \mathrm{p}(\lambda) d\left(\pi^{2} \lambda^{2}\right) \leqq E(\Lambda)
$$

by (4.5.3), since $\mathfrak{B}_{2}^{\dagger} \in \mathcal{T}\left(\alpha_{1}\right)$; and from the normalization (5.1.1) we see that

$$
\int_{0}^{\lambda_{0}} \mathrm{p}(\lambda) d\left(\pi^{2} \lambda^{2}\right)=G\left(\lambda_{0}\right)+\mathrm{O}\left(\lambda_{0}^{3}\right)
$$

We suppose that $\lambda_{0}<\Lambda_{2}$, and then, using (8.6.8) and (8.6.9), we have

$$
\begin{aligned}
I_{1} & =8 \int_{\lambda_{0}}^{\Lambda_{2}}\left[\int_{0}^{\Lambda} \mathrm{p}(\lambda) d\left(\pi^{2} \lambda^{2}\right)-\int_{0}^{\lambda_{0}} \mathrm{p}(\lambda) d\left(\pi^{2} \lambda^{2}\right)\right] \Lambda^{-3} d \Lambda \\
& \leqq 8 \int_{\lambda_{0}}^{\Lambda_{2}}\left[E(\Lambda)-G\left(\lambda_{0}\right)\right] \Lambda^{-3} d \Lambda+O\left(\lambda_{0}\right) .
\end{aligned}
$$

Also 


$$
\begin{aligned}
I_{2} & =8 \int_{\Lambda_{2}}^{\infty}\left[\int_{0}^{\Lambda} \mathrm{p}(\lambda) d\left(\pi^{2} \lambda^{2}\right)-\int_{0}^{\lambda_{0}} \mathrm{p}(\lambda) d\left(\pi^{2} \lambda^{2}\right)\right] \Lambda^{-3} d \Lambda \\
& \leqq 8 \int_{\Lambda_{2}}^{\infty}\left[E\left(\Lambda_{2}\right)-G\left(\lambda_{0}\right)\right] \Lambda^{-3} d \Lambda+\mathrm{O}\left(\lambda_{0}\right) .
\end{aligned}
$$

Let

$$
G_{1}(\Lambda)=\left\{\begin{array}{l}
G(\Lambda), 0 \leqq \Lambda<\Lambda_{2}, \\
G\left(\Lambda_{2}\right), \Lambda \geqq \Lambda_{2},
\end{array} E_{1}(\Lambda)=\left\{\begin{array}{l}
E(\Lambda), 0 \leqq \Lambda<\Lambda_{2}, \\
E\left[\Lambda_{2}\right], \Lambda \geqq \Lambda_{2} .
\end{array}\right.\right.
$$

Then substituting from (8.6.10), (8.6.11) and (8.6.12) into (8.6.7) we obtain the inequality

$$
\begin{aligned}
V_{2} & \leqq 8 \int_{\lambda_{0}}^{\infty}\left[E_{1}(\Lambda)-G_{1}\left(\lambda_{0}\right)\right] \Lambda^{-3} d \Lambda+O\left(\lambda_{0}\right) \\
& \leqq 8 \int_{\lambda_{0}}^{\infty}\left[G_{1}(\Lambda)-G_{1}\left(\lambda_{0}\right)\right] \Lambda^{-3} d \Lambda+8 \alpha_{1}+O\left(\lambda_{0}\right)
\end{aligned}
$$

by (4.5.1) and (4.5.2). A simple calculation now gives

$8 \int_{\lambda_{0}}^{\infty}\left[G_{1}(\Lambda)-G_{1}\left(\lambda_{0}\right)\right] \Lambda^{-3} d \Lambda=\frac{1}{2} \int_{\lambda_{0}}^{\Lambda_{2}}|\lg (2 / \lambda)| d\left(4 \pi^{2} \lg \lambda^{2}\right)$

$$
\begin{aligned}
& =2 \pi^{2} \lg ^{2} \lambda_{0}+4 \pi^{2} \lg 2 \cdot \lg \left(1 / \lambda_{0}\right) \\
& +\operatorname{sgn}\left(\Lambda_{2}-2\right) \cdot\left[2 \pi^{2} \lg ^{2} \Lambda_{2}+4 \pi^{2} \lg 2 \cdot \lg \left(1 / \Lambda_{2}\right)\right] \\
& +2 \pi^{2} \lg ^{2} 2 \cdot\left[1+\operatorname{sgn}\left(\Lambda_{2}-2\right)\right],
\end{aligned}
$$

where

$$
\operatorname{sgn} a=\left\{\begin{aligned}
1, & \text { if } \quad a \geqq 0, \\
-1, & \text { if } \quad a<0 .
\end{aligned}\right.
$$

and substituting from (8.6.14) into (8.6.13), we obtain

$$
\begin{aligned}
V_{2} \leqq & 2 \pi^{2} \lg ^{2} \lambda_{0}+4 \pi^{2} \lg 2 \cdot \lg \left(1 / \lambda_{0}\right) \\
& +\operatorname{sgn}\left(\Lambda_{2}-2\right) \cdot\left[2 \pi^{2} \lg ^{2} \Lambda_{2}+4 \pi^{2} \lg 2 \cdot \lg \left(1 / \Lambda_{2}\right)\right] \\
& +2\left[1+\operatorname{sgn}\left(\Lambda_{2}-2\right)\right] \pi^{2} \lg ^{2} 2+8 \alpha_{1}+O\left(\lambda_{0}\right) .
\end{aligned}
$$

8.7. Since $\mathbf{w}^{-1}\left(\mathfrak{B}_{2}^{\dagger}\right)$ is schlicht, and $\mathbf{w}^{-1}\left(\mathfrak{B}_{2}^{\dagger}\right) \subset \mathfrak{B}_{1} \subset \mathfrak{B}_{0}$, we see that $\mathbf{w}^{-1}\left(\mathfrak{B}_{2}^{\dagger}\right)$ is mean one-valent with excess 0 . Since

$$
\mathrm{V}\left[\mathbf{w}^{-1}\left(\mathfrak{B}_{2}^{\dagger}\right)\right] \leqq \mathrm{V}\left(\mathfrak{B}_{1}\right)=\pi^{2},
$$

we can choose a number $\Lambda_{1}, 0<\Lambda_{1} \leqq 2$ such that

$$
\mathrm{V}\left[\mathrm{w}^{-1}\left(\mathfrak{B}_{2}^{\dagger}\right)\right]=G\left(\Lambda_{1}\right),
$$

and a calculation similar to that of $\$ 8.6$ (but simpler) then gives 


$$
\begin{aligned}
V_{1} \leqq & 2 \pi^{2} \lg ^{2} \lambda_{0}+4 \pi^{2} \lg 2 \cdot \lg \left(1 / \lambda_{0}\right) \\
& -2 \pi^{2} \lg ^{2} \Lambda_{1}-4 \pi^{2} \lg 2 \cdot \lg \left(1 / \Lambda_{1}\right)+O\left[\lambda_{0} \lg \left(1 / \lambda_{0}\right)\right] .
\end{aligned}
$$

8.8. The theorem is now immediate. We suppose first that $0<\Lambda_{2}<2$. Then substituting from (8.6.15) and (8.7.2) into (8.5.2), and letting $\lambda_{0} \rightarrow 0$, we obtain

$$
2 \pi^{2}\left(\lg ^{2} \Lambda_{1}+\lg ^{2} \Lambda_{2}\right)+4 \pi^{2} \lg 2 \cdot \lg \left(1 / \Lambda_{1} \Lambda_{2}\right) \leqq 8 \alpha_{1}+2 \alpha_{2},
$$

and so $a$ fortiori

$$
\Lambda_{1} \Lambda_{2} \geqq \exp \left[-\left(4 \alpha_{1}+\alpha_{2}\right) / 2 \pi^{2} \lg 2\right] .
$$

If $\Lambda_{2} \geqq 2$, we have similarly

$$
2 \pi^{2}\left(\lg ^{2} \Lambda_{1}-\lg ^{2} \Lambda_{2}\right)+4 \pi^{2} \lg 2 \cdot \lg \left(\Lambda_{2} / \Lambda_{1}\right) \leqq 4 \pi^{2} \lg ^{2} 2+8 \alpha_{1}+2 \alpha_{2} .
$$

Either $\Lambda_{1} \Lambda_{2} \geqq 1$, or $\Lambda_{1}<1 / \Lambda_{2}$. In the latter case,

$$
\lg ^{2} \Lambda_{1}-\lg ^{2} \Lambda_{2} \geqq 0,
$$

and so (again a fortiori)

$$
\Lambda_{1} \geqq \frac{1}{2} \Lambda_{2} \cdot \exp \left[-\left(8 \alpha_{1}+2 \alpha_{2}\right) / 4 \pi^{2} \lg 2\right] \geqq \exp \left[-\left(4 \alpha_{1}+\alpha_{2}\right) / 2 \pi^{2} \lg 2\right] .
$$

Hence for all $\Lambda_{2}>0$,

$$
\begin{aligned}
\Lambda_{1} \Lambda_{2} & \geqq \min \left\{\exp \left[-\left(4 \alpha_{1}+\alpha_{2}\right) / 2 \pi^{2} \lg 2\right], 1\right\} \\
& =\exp \left[-\left(4 \alpha_{1}+\alpha_{2}\right) / 2 \pi^{2} \lg 2\right] .
\end{aligned}
$$

Finally

$$
\mathrm{V}\left[\mathrm{w}^{-1}\left(\mathfrak{B}_{2}^{\dagger}\right)\right] \cdot \mathrm{V}\left[\mathfrak{B}_{2}^{\dagger}\right]=G\left(\Lambda_{1}\right) E\left(\Lambda_{2}\right) \geqq G\left(\Lambda_{1}\right) G\left(\Lambda_{2}\right) .
$$

If $0<\Lambda_{2}<2$, then

$$
\begin{aligned}
G\left(\Lambda_{1}\right) G\left(\Lambda_{2}\right) & =\frac{1}{4} \pi^{4}\left\{\left[\lg 2-\lg \Lambda_{1}\right] \Lambda_{1}^{2}+\frac{1}{2} \Lambda_{1}^{2}\right\}\left\{\left[\lg 2-\lg \Lambda_{2}\right] \Lambda_{2}^{2}+\frac{1}{2} \Lambda_{2}^{2}\right\} \\
& \geqq(\pi / 16) \Lambda_{1}^{2} \Lambda_{2}^{2} .
\end{aligned}
$$

Secondly, if $2 \leqq \Lambda_{2}<4$, then $G\left(\Lambda_{2}\right) \geqq \frac{1}{4} \pi^{4} \Lambda_{2}^{2} \geqq \pi^{2}$, and so

$(8.8 .4) G\left(\Lambda_{1}\right) G\left(\Lambda_{2}\right) \geqq \pi^{2} G\left(\Lambda_{1}\right)=\left(\pi^{4} / 2\right)\left\{\left[\lg 2-\lg \Lambda_{1}\right] \Lambda_{1}^{2}+\frac{1}{2} \Lambda_{1}^{2}\right\} \geqq(\stackrel{4}{\pi} / 64) \Lambda_{1}^{2} \Lambda_{2}^{2}$.

Lastly, if $\Lambda_{2} \geqq 4$,

$$
\begin{aligned}
G\left(\Lambda_{1}\right) G\left(\Lambda_{2}\right)= & \left(\pi^{4} / 4\right)\left\{\left[\lg 2-\lg \Lambda_{1}\right] \Lambda_{1}^{2}+\frac{1}{2} \Lambda_{1}^{2}\right\} \\
& \left\{\left[\lg \Lambda_{2}-\lg 2\right] \Lambda_{2}^{2}+4-\frac{1}{2} \Lambda_{2}^{2}\right\} \\
\geqq & \left(\pi^{4} / 8\right)\left\{\left[\lg 2-\frac{1}{2}\right] \Lambda_{2}^{2}\right\}\left\{\Lambda_{1}^{2}\right\} \geqq\left(\pi^{4} / 64\right) \Lambda_{1}^{2} \Lambda_{2}^{2} .
\end{aligned}
$$

The theorem (apart from the restriction that $\mathrm{n}_{\mathfrak{B}_{2}}\left(0, \mu, \psi_{1}, \psi_{2}\right) \leqq 1$ ) follows from (8.8.1), (8.8.2), (8.8.3), (8.8.4) and (8.8.5). 


\section{THE RESTRICTION THAT $\mathrm{n}_{\mathfrak{B}_{2}}\left(0, \lambda, \psi_{1}, \psi_{2}\right) \leqq 1$}

\subsection{In $\$ 7$ we supposed that}

$$
\mathrm{n}_{\mathfrak{B}_{2}}\left(0, \lambda, \psi_{1}, \psi_{2}\right) \leqq 1 .
$$

We now indicate how this hypothesis may be removed. Since, however, the inclusion of all details would lengthen the paper considerably, we give only a sketch.

9.2. We proceed as follows. We define $\mathfrak{B}_{2}^{\dagger}$ as the subdomain of points $P\left(\lambda, \mu, \psi_{1}, \psi_{2}\right)$ of $\mathfrak{B}_{2}$ for which: (i) $\lambda>0$; (ii) there exists a pair of numbers $\left(\psi_{1}, \psi_{2}\right)$ and a path lying in

$$
\mathrm{E}\left[\arg w_{k}=\psi_{k},\left(w_{1}, w_{2}\right) \in \mathfrak{B}_{2},\left|w_{k}\right|>0\right]
$$

which connects (in the sense of $\S 7$ ) $P$ to a neighborhood of the point $\mathbf{w}(0,0)$. Let $\mathfrak{B}_{2}^{\text {t† }}$ be the subdomain of $\mathfrak{B}_{2}$ any point $P$ of which can be connected with $\mathbf{w}(0,0)$ along a path the transform of which in $\mathfrak{B}_{1}$ is contained in

$$
\mathrm{E}\left[\arg w_{k}\left(z_{1}, z_{2}\right)=\psi_{k},\left|z_{k}\right|<1\right]-\mathrm{E}\left[\left|z_{1}\right|=0\right]-\mathrm{E}\left[\left|z_{2}\right|=0\right] .
$$

Then $\mathfrak{B}_{2}^{\dagger} \subset \mathfrak{B}_{2}^{\dagger \dagger}$. We have

LeMma 6. If $\mathfrak{B}_{2}^{\dagger} \in \mathcal{T}\left(\alpha_{1}\right)$, then $\mathfrak{B}_{2}^{\dagger}$ and $\mathfrak{B}_{2}^{\dagger \dagger}$ are identical.

In fact, let $\mathrm{n}^{\dagger \dagger}\left(\lambda, \mu, \psi_{1}, \psi_{2}\right)$ be the number of times $\mathfrak{B}_{2}^{\dagger \dagger}$ covers the point $\left(\lambda, \mu, \psi_{1}, \psi_{2}\right)$. Then Lemma 6 is plainly equivalent to the assertion that

$$
\mathrm{n}^{\dagger \dagger}\left(0, \mu, \psi_{1}, \psi_{2}\right) \leqq 1
$$

Suppose now that a point $P_{0}$ on $\lambda=0$ were covered by $\mathfrak{B}_{2}^{\text {t† }}$ twice. Then there is a path

$$
\mathfrak{r}^{1}=\mathrm{E}\left[r_{1}(s) e^{i \psi_{1}^{0}}, r_{2}(s) e^{i \psi_{2}^{0}}, 0 \leqq s \leqq 1\right],
$$

with

$$
\left[r_{1}(0) e^{i \psi_{1}}, r_{2}(0) e^{i \psi_{2}}\right]=P_{0}\left[r_{1}(1), r_{2}(1)\right]=\mathrm{w}(0,0),
$$

lying, except for its end points, in the portion of $\mathfrak{B}_{2}$ for which $\lambda>0$. Hence there is a P $>0$ for which the "tube"

$$
\Re=\underset{\left(\omega_{1}, \omega_{2}\right) \in \mathbf{r}^{1}}{S}\left[\left|w_{1}-\omega_{1}\right|^{2}+\left|w_{2}-\omega_{2}\right|^{2} \leqq \mathrm{P}^{2}\right]
$$

is contained in $\mathfrak{B}_{2}$. Since $\Re$ lies in a finite region of space, there is an $\epsilon, \epsilon>0$ such that the set

$$
\mathrm{E}\left[\left(r_{1}(s)+\epsilon_{1}\right) e^{i\left(\psi_{1}+\epsilon_{2}\right)},\left(r_{2}(s)+\epsilon_{3}\right) e^{i\left(\psi_{2}+\epsilon_{4}\right)},\left|\epsilon_{j}\right|<\epsilon\right]
$$

is contained in $\Re$. If

$$
\mathrm{n}^{\dagger}\left(\lambda, \mu, \psi_{1}, \psi_{2}\right)
$$

is the number of times $\mathfrak{B}_{2}^{\dagger}$ covers the point $\left(\lambda, \mu, \psi_{1}, \psi_{2}\right)$, we have, therefore 


$$
\mathrm{n} \dagger\left(\lambda, \mu, \psi_{1}, \psi_{2}\right)>1
$$

in a four-dimensional domain

$$
\begin{aligned}
& \mathfrak{N}\left(\lambda_{1}\right)=\mathrm{E}\left[0<\lambda<\lambda^{(1)}, \mu^{(1)}<\mu<\mu^{(2)},\right. \\
& \left.\psi_{1}^{(1)}<\psi_{1}<\psi_{1}^{(2)}, \psi_{2}^{(1)}<\psi_{2}<\psi_{2}^{(2)}\right] .
\end{aligned}
$$

From the normalization (5.1.1) we now have

$$
\begin{aligned}
\mathrm{V}\left[\mathfrak{B}^{+}(\Lambda)\right]= & \frac{1}{16} \int_{-\pi}^{\pi} \int_{-\pi}^{\pi} \int_{0}^{\Lambda} \int_{-\infty}^{\infty} \mathrm{n} \dagger\left(\lambda, \mu, \psi_{1}, \psi_{2}\right) \frac{d \mu}{\left(\lambda^{2}+\mu^{2}\right)^{1 / 2}} d\left(\lambda^{2}\right) d \psi_{1} d \psi_{2} \\
\geqq & \frac{1}{16} \int_{-\pi}^{\pi} d \psi_{1} \int_{-\pi}^{\pi} d \psi_{2} \int_{0}^{\Lambda} d\left(\lambda^{2}\right) \int_{-\left(1-\lambda^{2} / 4\right)}^{\left(1-\lambda^{2} / 4\right)}\left(\lambda^{2}+\mu^{2}\right)^{-1 / 2} d \mu \\
& +\frac{1}{16} \iiint \int_{\Re(\lambda)}\left(\lambda^{2}+\mu^{2}\right)^{-1 / 2} d \mu d\left(\lambda^{2}\right) d \psi_{1} d \psi_{2}+\mathrm{O}\left(\Lambda^{3}\right) \\
= & \mathrm{V}\left[\mathfrak{B}_{0}(\Lambda)\right]+\frac{1}{16} \iiint \int_{\Re(\lambda)}\left(\lambda^{2}+\mu^{2}\right)^{-1 / 2} d \mu d\left(\lambda^{2}\right) d \psi_{1} d \psi_{2} \\
& +\mathrm{O}\left(\Lambda^{3}\right)>\mathrm{V}\left[\mathfrak{B}_{0}(\Lambda)\right]+\alpha_{1} \Lambda^{3} F(\Lambda),
\end{aligned}
$$

for a sequence of $\Lambda$ tending to zero. This contradicts (4.5.3), and proves Lemma 6.

9.3. Finally, by Lemma 6 we see that for the mapping (6.2.1) there exists a $\delta=\delta(\rho)$ such that

$$
\mathrm{n} \dagger\left(\lambda, \mu, \psi_{1}, \psi_{2}\right) \leqq 1,
$$

if $0<\lambda<\delta$.

We now apply the method of $\S 7$ to $\mathfrak{S}_{2}^{\dagger 2}\left(\psi_{1}, \psi_{2}\right)$ without introducing $\mathfrak{S}_{2}^{2}\left(\psi_{1}, \psi_{2}\right)$. Apart from minor complications, the proof then proceeds substantially as written out above.

\section{BiBLIOGRAPHY}

\section{Stefan Bergman}

1. Über eine in der Theorie der Funktionen von zwei komplexen Veränderlichen auftretende unitäre Geometrie, Proceedings, Akademie van Wetenschappen, Amsterdam, vol. 36 (1933), pp. 307-313.

2. Sur quelques propriêtés des transformations par un couple des fonctions de deux variables complexes, Rendiconti delle R. Accademia Nazionale dei Lincei, (6), vol. 19 (1934), pp. 474-478.

3. Über eine Abschätzung von meromorphen Funktionen zweier komplexen Veränderlichen in Bereichen mit ausgezeichneter Randfläche, Travaux de l'Institut Mathématique de Tbilissi, vol. 1 (1937), pp. 187-204.

4. Theory of pseudo-conformal transformations and its connection with differential geometry, Notes of lectures delivered at the Massachusetts Institute of Technology, 1939-1940 (available at the Brown University library).

5. Lectures on conformal mapping of multiply-connected domains (Partial Differential Equations, Advanced Topics), Publication of Brown University, 1941. 
6. Sur les Fonctions Orthogonales de Plusieurs Variables Complexes avec les Applications d̀ la Théorie des Fonctions Analytiques, Interscience Publishers, New York, 1941.

\section{A. Bermant}

1. Remarque sur le lemme de Schwarz, Comptes Rendus de l'Académie des Sciences, Paris, vol. 207 (1938), pp. 31-33.

G. M. Golusin

1. Einige Überdeckungssatze für die im Kreise regulären Funktionen, Recueil Mathématique, (n.s.), vol. 2(44) (1937), pp. 617-619.

W. F. OsGood

1. Lehrbuch der Funktionentheorie, vol. 2, part 1.

D. C. SPENCER

1. On finitely mean valent functions, Proceedings of the London Mathematical Society, (2), vol. 47 (1941), pp. 201-211.

2. On an inequality of Grunsky, Proceedings of the National Academy of Sciences, vol. 26 (1940), pp. 616-621.

3. On a theorem of Rengel, Journal of Mathematics and Physics, vol. 20 (1941), pp. 118123.

Brown UNIVERSITY, Providence, R.I.

Massachusetts Institute of Technology, Cambridge, Mass. 